\title{
The Open Mind: A Phenomenology
}

\author{
Josh Adler \\ The Shantigar Foundation, Rowe, MA, USA \\ Email: Josh@TreeMedia.com
}

How to cite this paper: Adler, J. (2021). The Open Mind: A Phenomenology. Open Journal of Philosophy, 11, 249-291. https://doi.org/10.4236/ojpp.2021.112019

Received: March 11, 2021

Accepted: May 9, 2021

Published: May 12, 2021

Copyright (c) 2021 by author(s) and Scientific Research Publishing Inc. This work is licensed under the Creative Commons Attribution International License (CC BY 4.0).

http://creativecommons.org/licenses/by/4.0/ 
in order to interpret and communicate our sensations, thoughts, and complete experiences, therefore reveals a radically multidimensional bio-geometry and biolinguistics based on a field dynamics, which Eco's work constructs. Via an aesthetic information theory, Eco describes this phenomenon as, "a practical level of poetics that acts as programmatic projects for creation." The writings of Rumi, Husserl, Dewey, Emerson, Merleau-Ponty, Hegel, and others confer depth to the emergent mechanics of perception found throughout biotic systems. Eco's ideas thereby provoke discourse on the role of openness within recent theoretical works by Jeremy England, Anirban Bandyopadhyay, Robert Lanza, Deepak Chopra, Ervin Laszlo, Giulio Tononi, Stuart Kauffman, Walter J. Freeman, Robin Carhart-Harris, Mark P. Mattson, Robert R McCrae, Selen Atasoy, Katherine Peil, Terence Deacon, and David Chalmers. Unlike models of consciousness that arise from closed, local computation, I argue that the phenomenon of open mindedness unifies cultural and scientific concepts of consciousness as life's critical integrative force.

\section{Keywords}

Biolinguistics, Philosophy of Mind, Phenomenology, Consciousness Systems Theory, Field Dynamics, Openness, Aesthetic Information

\section{Introduction}

"Open mind" is a contemporary concept rife in popular cultural memetics since at least the late 1950s, when "father of the atomic bomb" J. Robert Oppenheimer applied it to governance, as "an indispensable, perhaps in some ways the indispensable, element in giving meaning to the dignity of man...." Later in the same article Oppenheimer concludes, "The style, the perceptiveness, the imagination, and the open-mindedness with which we need to conduct our [governmental] affairs can only pervade.... if they are a reflection of a deep and widespread public understanding" (Oppenheimer, 1949). M. Rokeach and his colleagues in "The Open and Closed Mind" followed soon after by arguing sociologically that, "a rigid cognitive organization of attitudes and values leads to predictable social consequences, including prejudice and authoritarian submission" (Rokeach, 1960; McCrae, 1996).

First published in 1962, The Open Work, by Umberto Eco, provides a poetics-based critique of "openness," as a crucial developmental aspect of contemporary art's engagement with cultural formativity (Eco, 1989). He writes, "In art, the individual forms for the sake of forming, thinks and acts in order to form... form is a structured object uniting thought, feeling, and matter in an activity that aims at the harmonious coordination of all three and proceeds according to the laws postulated and manifested by the work itself as it is being made... a form, once it has reached completion and autonomy, can be seen as perfect only if it is dynamically considered. Aesthetic contemplation is this active consideration that retraces the process which gave life to form... But since the fact of form 
opens it up to an infinity of different perspectives, the process which actualizes itself as form also realizes itself in the continuous possibility of interpretation" (Eco, 1989). Eco's understanding here of aesthetic contemplation as a significant process in consciousness' dynamic organization of a perceptual continuum outlines its faculty as the primary receptive interpreter between indeterminate and deterministic neurological fields. For instance, the process of "harmonious coordination" he describes can be challenged by competing views that result in "cognitive dissonance" if the interpreter is unable to remain open to co-emergently different, yet viable, perspectives. His position therefore supports an understanding of open-mindedness as a compositional phenomenon that allows consciousness to combine into ever more complex activity, as well as a creative source of new physical possibilities.

More recently in 1989, psychologist Robert R. McCrae conducted the study, "Social Consequences of Experiential Openness," which revealed the deep social and political implications of individual trends in intrapsychical and interpersonal aspects of openness. He writes, "Openness is manifested in 'the breadth, depth, and permeability of consciousness, and in the recurrent need to enlarge and examine experience"' (McCrae, 1996). Within the domain of what Eco deems, "structural homologies," McCrae includes within the phenomenology of openness: processes of intuition, thin mental boundaries, and typical intellectual engagement. "Openness," he writes, "is a psychological construct centered on intellectual engagement and aesthetic experience and only secondarily reflected in social and political attitudes." Overall his research deepens the perception of openness as "a fundamental way of approaching the world that affects not only internal experience but also interpersonal interactions and social behavior" (McCrae, 1996). Current psychological investigation is now tracing the "genetic and phenotypically distinct" factors of Openness' personality dimensions, which relate cognitive engagement to creativity and intellect as "reflecting the tendency toward cognitive exploration” (Kaufman et al., 2015).

In order to provide an integrative analysis of previous cultural and psychological studies of openness with current biological models, this work will build a phenomenologically based Conscious Systems Theory (CST) upon various, even disjointed, correlations between mechanics, dynamics, and aesthetics in conscious systems. The discussion proposes a common field dynamics, which establishes a coherent harmonic structure capable of transmitting information within observed physical and mental phenomena. This "Mechanics, Dynamics, Aesthetics (MDA)" methodology entails a great deal of cross-disciplinary aggregation, and was generously introduced to me by Jerrold McGrath, during a program at the Banff Centre. By applying this discipline for framing systems from game theory, my research aims to effectively examine and constitute the associations between current theories of quantum biology and cultural semiotics into a practical linguistics of consciousness studies.

One of the plausible challenges against shifting out of the primarily materialist reductionist framework of current consciousness studies is a critical resistance to 
accept and apply ontological arguments from cultural studies to hard sciences. Such interdisciplinary opposition seems to be the norm, and rightfully often considered an ill-fitting endeavor. In Lacan Between Cultural Studies and Cognitivism, Slavoj Zizek advocates against the temptation that lies within cultural studies to mix-and-match paradigms from hard sciences. He writes, "...the moment one wants to provide an ontological account of quantum physics (what notion of reality fits its results), paradoxes emerge... cultural studies denounce the very attempt to draw a clear line of distinction between, say, true science and prescientific mythology" (Zizek, 2002). However, in the emerging science of consciousness, linguistics of preceding philosophical hegemony may play an obstructive role in the capacity of the field to recognize key aspects of its own significance. As Eco points out: "While science, today, limits itself to suggesting a probable structure of things, art tries to give us a possible image of this new world, an image that our sensibility has not yet been able to formulate, since it always lags a few steps behind intelligence-indeed, so much so, that we still say the sun 'rises' when for three centuries we have known it does not budge" (Eco, 1989).

One rife epistemological example for expanding our biolinguistic understanding is the perceived phenomenon of "security". As a driver of both pain and pleasure principles throughout living systems, conscious attitudes towards "security" reveals an opportunity to frame the thermodynamic and harmonic dance of variabilities that order attractors and qualia transfers (or imprinting), via regulatory pathways of homeostasis. The bounds of security percepts compel biodynamic effects as complex as geopolitics in human affairs, and as basic as bacterial binary fission. In Drosophilia embryo, development patterns are secured by waveforms clearing genetic "dead zones" through transcript patterns that "yield progressively finer grained positional information" (Kauffman \& Goodwin, 1990). Exploring, questioning, and expanding our scientific and cultural consciousness of such a biolinguistic phenomenon opens adjacent organizational possibilities, not only in terms of meaning, but also by recalibrating improbable qualia producing combinations of awareness and attention (Kauffman, 2012). There are vital questions in understanding how "security" affects biological predictive possibilities in the generation of feed forward loops that supercausally impact experiential properties and probabilities by primitive "Merge" operations (Chomsky, 2007; Vannini, 2009). For instance, how might perceptual planning alter the actualities of reality across genetic regulatory networks? Investigating these deeper structures of language, according to Chomsky, shows how “... acquisition of language involves not just a few years of experience and millions of years of evolution, yielding the genetic endowment, but also principles of neural organization that may be even more deeply grounded in physical law" (Chomsky, 2007). Furthermore, Chomsky resonates with Eco in commenting, "An elementary fact about the language faculty is that it is a system of discrete infinity." But how does open mindedness impact evolutionary adaptation via cognitive engagement? How open can the biotic agents of attention or awareness 
be while corporally bound, and how does its dynamic positioning during neurological imprinting affect the quality of perception in living systems?

Crucial to a discursive recontextualization of consciousness as biology's key compositional force is an enhanced understanding of the nature of "information" to implicate physics as much as semiotics, or computation (Kauffman, 2012). Often regarded in contemporary culture as equivalent to "data," which refers merely to an algorithmic, reductive functionality, information's definition here must be expanded to include all aspects of biological perception, such as sensation, and the poetic aspects of non-algorithmic, aesthetic interpretation; noted by both Roger Penrose and Umberto Eco as key indicators of consciousness's true multifaceted significance as a primary force of biological integration, agency, and creation. Therefore, the term information should be regarded in this study as any stimulus that can be biologically communicated, organized, or interpreted. By suggesting such a biolinguistically "generative" syntactic shift, researchers may find room to move their inquiries towards a more dynamic framework, rife with greater articulation for both physical and experiential Neural Correlates of Consciousness (NCCs). Joachim Keppler is one quantum physicist who is effectively taking up this call to "re-code" our linguistic approach to understanding the mechanics of consciousness as a phenomenal extraction from qualia producing "information states." His work with Stochastic Electrodynamics (SED) introduces the brain as dynamically "coupled"; or crudely put, somewhat like modern "Wi-Fi" devices with a fundamental background field from which consciousness adapted to interpret useful signals, such as food sources or music, from noise. More on Keppler's work to follow.

In further research of this linguistic reassessment, John Wheeler, J. Keppler, E. Laszlo, and others have argued in favor of viewing the Zero-Point Field (ZPF) as a "plenum" rather than the more prevalent conception of a "vacuum." In support of their arguments, current studies reveal that our universe is filled with enough energy waves, light, gravitation, and matter (including dark forms) to belie any claims of physical "emptiness" implied by correlating the ZPF with a vacuum. A reflection of this "empty cosmos" trope also plays out in traditional Buddhist understanding of self and subjectivity. Here the conversation also feels ripe for disruption due to developments in biolinguistics. Zizek observes the Lacanian concept of subjectivity as an ongoing process of misidentification operating in a continuous flux of formativity in relation to a paradigm of systematic attractors. Therefore the "self" would be better represented and evaluated in terms of its receptivity to information processing and communications abilities, or in other words "openness," which can potentially speak more comprehensively to life's evolution towards cognitive complexity, via primal hedonistic pathways of social and cultural engagement (Peil, 2016).

Here is a crucial consideration: Were our subjectivities, or the perceptual faculties of any living organism, based on a fundamentally "closed" phenomenology, much of the communicativity of biological systems would simply not exist. 
In a closed, or fundamentally narrow system, plants would not be able to absorb light into energy, cells would not scale into organs, language would never develop, imagination could not grasp the impossible to constitute new possibilities, and many other emergently integrative aspects of our reality would keep life isolated into islands of siloed feedback loops. Imagining such a universe raises the specter of dualistic thinking in a way that reveals how far afield our interdependent web of life really is from a purely materialistic physical model wherein brains are machines, and consciousness relegated to a purely deterministic closed-circuit apparition. Were we inhabitants of such a closed world, no music would be possible, as there would be no means to communicate compositionally, no awareness to share, and thereby no self-determining means to transmit our experience. It is the basic openness of our reality-a fundamental phenomenon providing the field, context, or background for dynamic combinatorial interactions to create unprecedented compositional states, forms, and complexity-that allows for local and non-local coherence between quantum and standard models, self-organizational synchrony in evolutionary systems, and the integrated receptivity and expressiveness of consciousness. Until now this perspective has gone largely unstudied in biological terms.

Kafatos, Tanzi, and Chopra expand Eco's ethos when they recommend, "In our view, it may well be that the subject-object dichotomy is false to begin with and that consciousness is primary in the cosmos, not just an epiphenomenon of physical processes in a nervous system" (Kafatos, Tanzi, \& Chopra, 2011). Therefore, perhaps we can finally distend any epistemological need for a "ghost in the machine" by adopting a theoretical consideration of the "conscious system" into: a continuous system of interconnected, living systems, nested within the finely tuned quantum plenum (i.e., ZPF) across all scales of order in varying, dynamic states of openness and localities to each other. Within such a consideration, an individual organism's consciousness, which is regulated by its sense of "openness," would constitute its veritable agency, (i.e., its force, or potential impact) within the total system, which correlatively generates its unique inner life.

Openness, unlike the semiotics of emptiness, can be evaluated in terms of the ability of conscious systems to process "aesthetic information." Aesthetic information, a term provided by Eco in counterpoint to quantitative information, perhaps provides the "non-algorithmic" neuro-linguistic potency suggested by the qualia geometry of IIT, and longed for by Roger Penrose in his investigation into the mathematical indicators of "free-will" (Eco, 1989; Penrose, 1999; Tono$\mathrm{ni}$, 2008). In contrast to quantitative information that "consists in drawing as many suggestions as possible out of a totality of signs with all the personal reactions that might be compatible" within an established system, Eco unveils the richness of aesthetic information as consistent in "referring the results drawn from the former type [quantitative information] back to their original organic qualities, in seizing, behind the suggestive wealth we exploit, a conscious organization, a formative intention, and in enjoying this new awareness. This awareness of the project that underlies the work will, in turn, be another inexhaustible 
source of pleasure and surprise," for all intents and purposes describing the accrual of meaning and agency attributed to an open, complex, and conscious system wherein interrelated coherent fields and organizational compounds can communicate through dynamic responsiveness.

As an example of an open system dependent on the perception of aesthetic information, Eco puts forward Joyce's Finnegan's Wake.

In Finnegan's Wake we are faced with an even more startling process of "openness": the book is molded into a curve that bends back on itself, like the Einsteinian universe. The opening word of the page is the same as the closing word of the last page of the novel. Thus, the work is finite in one sense, but in another sense it is unlimited. Each occurrence, each word stands in a series of possible relations with all the others in the text. According to the semantic choice that we make in the case of one unit, so goes the way we interpret all the other units in the text. This does not mean that the book lacks specific sense. If Joyce does introduce some keys into the text, it is precisely because he wants the work to be read in a certain sense. But this particular "sense" has all the richness of the cosmos itself (Eco, 1989).

We might substitute the term "work" for "system" from Eco's writings, nonetheless, his argument compellingly proclaims, "thus, in the dialectics [perhaps 'oscillations' in quantum biological terms] between work and openness, the very persistence of the work is itself a guarantee of both communication and aesthetic pleasure. Not only are the two values intimately connected, but each implies the other-which is certainly not the case with a conventional message such as a road sign, where the act of communication exists without any aesthetic effect and exhausts itself in the apprehension of the referent, without ever inducing us to return to the sign to enjoy the effectiveness of its message in the way it is formally expressed. 'Openness,' on the other hand, is the guarantee of a particularly rich kind of pleasure that our civilization pursues as one of its most precious values, since every aspect of our culture invites us to conceive, feel, and thus see the world as possibility." The semantic awareness that Eco imbues to our experience of openness suggests the degree of biolinguistics necessary to discuss the processes whereby even the most purely "internal" aspects of consciousness find correlative material expression. By establishing a poetics open to qualia-producing aesthetic interpretations, Eco shows how conscious organization can transform indeterminate thought, emotion, or sensation into emergent physical representation, action, and behavior. In much the same way, German researcher Joachim Keppler models transference of information states in quantum systems based on SED coupling, which allows particular systems to parsimoniously acquire their physical properties by the same means from which they acquire their phenomenal qualities (Keppler, 2016). In earlier work, Keppler explained, "the components of every physical system interact permanently and unavoidably with the zero-point field (ZPF), thus acquiring a stochastic motion 
and behaving as stochastic oscillators. As long as a system is sufficiently shielded against thermal noise and the ZPF is the dominating driving force, the energy exchange between the system components and the ZPF can reach equilibrium states where the average power absorbed by the system compensates exactly the average radiated power" (Keppler, 2013). More recently, Keppler has convincingly theorized that, "every quantum system is a conscious system, with the dynamic variability of a system determining the accessible spectrum of conscious states." Continuing, he argues that the complexity of conscious states arises from a system's openness to the spectrum of consciousness' all-pervasive substrate (i.e., the ZPF), which is also a phenomenological field discussed at length by Maurice Merleau-Ponty, and which I refer to craftily as "the Outernet". "Rather", writes Keppler, "evolution has brought forth increasingly complex quantum systems that rely on a universal and intelligible mechanism on the basis of which they are able to extract increasingly complex phenomenal qualities from the ubiquitous field of consciousness" (Keppler, 2016). For humans the primary extraction instrument is the brain, which recognizes information through a process that Hameroff, echoing Eco's semiotic mind, has both grandly and efficaciously interpreted as a means of "orchestration." All parties are supported by many years of research corroborating long-range coherence in the brain, which associates synchronization in the beta and gamma frequencies with NCC's of various conscious states (Keppler, 2013, 2016). Figure 1 below illustrates Keppler's model of the brain as a highly specialized filter of consciousness.

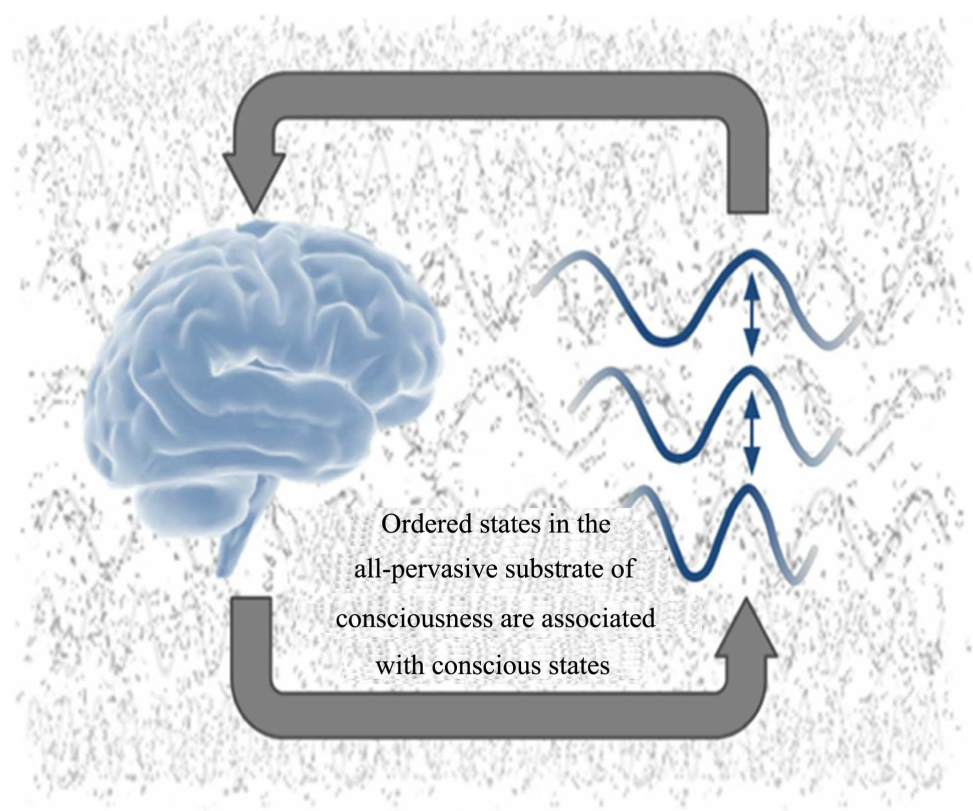

Figure 1. Joachim Keppler's hypothesis of a continuous interaction, via SED coupling, between the brain, and an all-pervasive background field which acts as the substrate of consciousness (Keppler, 2016. On the Universal Mechanism Underlying Conscious Systems and the Foundations for a Theory of Consciousness. Open Journal of Philosophy, 6, 346-367. doi: 10.4236/ojpp.2016.64034.). 
In the case of Finnegan's Wake, Eco implies that through consciousness's “intimate" exchanges between work and reader, respectively the non-local and local aspects of the same system, physical significance forms amid a phenomenologically fundamental continuum of "open-mindedness," including in its perception the communication of aesthetic interpretation, rather than merely deterministic results based on computation. The text acts as an attractor for the brain, which collapses the conscious system in moments of critical integration, while oscillating its openness between ordered and disordered phases to generate a poetics of dynamic variability based on cognitive harmonics.

\section{The Infinite at the Core of the Finite (How Do Expansions in Consciousness Adapt Living Systems?)}

In his discussion of the similarities and convergences within linguistic systems of an "open work", Umberto Eco writes:

What is at stake is a convergence of new canons and requirements, which the forms of art reflect by way of what we could term structural homologies. This need not commit us to assembling a rigorous parallelism-it is simply a case of phenomena like the "work in movement" simultaneously reflecting mutually contrasted epistemological situations, as yet contradictory and not satisfactorily reconciled. Thus, the concepts of "openness" and dynamism may recall the terminology of quantum physics: indeterminacy and discontinuity. (Eco, 1989)

In speaking compositionally (i.e. capable of constituting new possibilities via pattern discovery or recombination) about the "reciprocal play of problems" within the engagement of complex systems of awareness with art forms, Eco finds, "The possibilities which the work's openness makes available always work within a given field of relations." A quantum biological implication of Eco's compositional concept of openness and dynamism, involves living organisms' diverse abilities to organize the infinite variability of experience through communicable methods of aesthetic information.

One example is the pattern recognition process of our brain's neurons, as they consciously navigate the field of our sensory awareness in the interplay of attentional behaviors. Pattern recognition starts with special neurons called "control neurons" that unconsciously map potentially interesting parts of a scene. When an "attractor" catches someone's attention, the control neurons make a "window" that focuses around the subject of interest by strengthening the synaptic connections that lead to other parts of the brain. As one's attention increases, the control neurons balance the flow of memory feedback and visual information to interpret stimulation (Olshausen et al., 1993).

Husserl, with a prescient sense of control neurons writes:

Each state of consciousness implies the existence of a horizon which varies with the modification of its connections together with other states, and also 
with its own phases of duration... In each external perception, for instance, the sides of the objects which are actually perceived suggest to the viewer's attention the unperceived sides which, at the present, are viewed only in a nonintuitive manner and are expected to become elements of the succeeding perception. This process is similar to a continuous projection which takes on a new meaning with each phase of the perceptive process. Moreover, perception itself includes horizons which encompass other perceptive possibilities, such as a person might experience deliberately the direction of his perception, by turning his eyes one way instead of another, or by taking a step forward or sideways, and so forth (Eco, 1989).

As an example of biological perception's ability to involve the nonlocal absorption, integration, and transmission of "OR moments," encoded by discontinuities in space-time curvatures, gravitational dynamics, symmetries, and material states in resonance with information processing forms in the fine-scale structure of the universe; the role of control neurons in perception's integration of Stuart Kauffman's "adjacent possibilities” can account for even weak force organization of "proto-conscious" events into qualia producing architectures via stochastic electrodynamics (SED) (Penrose \& Hameroff, 2011). As Kauffman points out when interpreting the causal and acausal possibilities of two people meeting for coffee-just because one coffee shop closes, doesn't mean another across town won't be open. Therein he reveals how consciousness enables "changing actuals to change what is possible" (Kauffman, 2016).

Living systems are those that have evolved dynamic sensitivities that are "open" to receiving possible moments of awareness as they emanate from collapses in the fine scale or superstructure of the universe, which convert "proto-conscious" signals into SED information states. When faculties of attention reach a criticality of engagement, organisms make neurological imprints of aesthetic information, "what Penrose termed 'non-computable Platonic values' embedded in fundamental space-time geometry" (Hameroff, 2014). Keppler supports Penrose's description by adding:

It is widely accepted that consciousness is associated with long-range coherence in the brain, particularly with synchronized activity in the gamma frequency band. In more detail, new results suggest that "discrete moments of perceptual experience are implemented by transient gamma-band synchronization of relevant cortical regions, and that disintegration and reintegration of these assemblies is time-locked to ongoing theta oscillations" (Doesburg et al., 2009). Moreover, it was found that gamma synchrony shows up not only during attention to an external stimulus, but also in altered states of consciousness, such as meditation and REM sleep.

As for the characteristics of the gamma oscillations, a time-frequency analysis of the local field potentials (LFP) revealed that "the source of gamma-band peaks is of stochastic nature" (Burns et al., 2010) and that "gamma activity is indistinguishable from filtered noise" (Burns et al., 2011). Hence, 
gamma activity cannot be understood on the basis of deterministic network models. Rather, noise seems to play an essential role in the generation of gamma synchrony, so that in a realistic model the brain should be "viewed as a resonant stochastic oscillator" (Burns et al., 2010). Furthermore, also experiments investigating stochastic resonance (SR) within and between brain areas imply that "SR-mediated neural synchronization is a general mechanism of brain functioning" (Ward et al., 2006) and that "noise could play a fundamental role in biological information processing" (Keppler, 2013).

Pairing Keppler's understanding of quantum cognitive feedback dynamics in informational processing with the compositional qualia geometrics of IIT proposed by Giulio Tononi, suggests how consciousness's role plays out transactionally in the interdependent phenomenal fields of possibility, probability, awareness, attention, and experience (Keppler, 2016; Tononi, 2008). The first two form what Merleau-Ponty describes as the perceptual fields, and the latter three he describes as the sensorial fields (Merleau-Ponty, 2014).

This dynamic, yet harmonic, field structure therefore suggests a "bootstrap" approach to understanding how consciousness might universally weave through quantum processes of complex systems both micro and macro cosmically (Wolchover, 2017). Within living systems, the ubiquitous access to these background signals means that self-organizing information can plausibly be represented across entropic discontinuities via stochastic resonance (SR) processing to drive biological development of agency in close correlation to the thermodynamic work energy, and consequently "formativity," thereby driving reproduction (Perunov, Marsland, \& England, 2016). In this view, a plant that is open to processing energetic dynamics of light from the sun through photosynthesis, corresponds to the same long-range coherence of SR physical processes described by Hameroff's studies of microtubule organization of qualia within neurons. Remember: Eco confers that openness is a phenomenon of conscious organization that "locates the infinite at the very core of the finite" and "invites us to conceive, feel, and thus see the world as possibility" (Eco, 1989).

\section{Conscious Systems Theory (CST)}

By grounding its mechanics in a dynamic network of harmonic phenomenological fields (i.e., an Outernet), a Conscious Systems Theory (CST) proposes a non-panpsychic view of consciousness's operation. This hypothesis arrives at a view similar to IIT's "here, there, but not everywhere" approach, which characterizes consciousness as, "a fundamental property possessed by physical systems having specific causal properties. It predicts that consciousness is graded, is common among biological organisms and can occur in some very simple systems. Conversely, it predicts that feed-forward networks, even complex ones, are not conscious, nor are aggregates such as groups of individuals or heaps of sand" (Tononi \& Koch, 2015). A CST also considers the qualitative bonding aspect 
running through a "Quantum Underground," which Penrose describes as a realm where, "Individual particles then do not have 'states' on their own, but exist only in complicated 'entanglements' with other particles, referred to as correlations" (Penrose, 1999). As life's integrative force, consciousness co-relates its fundamental causes with its quantum effects, by existing as an aesthetically-based recombinator field phenomenon, featuring emergent, dynamic, multidimensional harmonic bonding (i.e., tunable) agency within all levels of order, that forms into living, embodied, self-organized, self-determining, sustained, occurrences with measurable internal resonance and radiance processes-including the inner (albeit open) life of the mind with attractor feedback loops that organize cognition.

Conscious Systems Theory (CST) utilizes the iterative, quantitative, and qualitative formal approach of MDA analysis to chunk together systems of thought the way the human brain chunks together phone numbers. Via the interrelatedness of a common field dynamics between aesthetics and mechanics responsive to opening and closing biofeedback (Hunicke, LeBlanc, \& Zubek, 2004). As dynamics are integrated throughout the system via events of collapse, consciousness regenerates its own coherence through perceivable aperiodic projections of space and time.

By allowing for aesthetic harmonics in conscious systems, CST recognizes the constitutive (i.e., mechanical) underpinnings of the co-relative variance in dynamically acquired agents, or subjects, to remain "open" or "closed" in terms of probabilistic and perceptive feedback, dependent on local biphasic cognitive processes of awareness, attention, and qualia. Generally, the basis of agency in conscious systems is their ability to choose to "stay open" to adversity, suffering or pain despite experiencing loads of discomfort, perturbations, or unpreferable states, or vice versa with regards to pleasurable experiences. Either way, closure leads to habit limiting behavior in living systems. Meanwhile, adaptive benefits of responsiveness gleaned from "staying open," are proven to allow for the development of self-modifiable, constitutional, creative, or mindful attractor principles based on Eco's understanding of "appreciation," which phenomenologically and neuro-correlatively requires less energy or reward potential to fire, yet permeates into much higher and subtler orders at play within a conscious system. This concurs with Hameroff's attitude of the "quantum pleasure principle," which cogently argues that life and the brain have evolved "to feel good" (Hameroff, 2015).

Challenging circumstantial environmental events, such as unpredictable changes in weather patterns, can often subvert biologically predictive assumptions; just as perceptual conscious behavior, such as taking an umbrella in case of rain, can also offset cognitive limitations. Therefore, creativity in the face of stochastic possibilities provides adaptively rewarding feed forward phenomena in the development of conscious systems, including intrapsychic phenomena (McCrae, 1996; Kaufman et al., 2015). These considerations suggest that "emergent "adaptive" resonance in the system" is correlated (both in terms of energy 
and information) to the system's openness to interpreting frequencies of driving field harmonics despite noise, interference, or perturbation during absorption (Perunov, Marsland, \& England, 2016). As a conscious perceiver remains more open more willfully, as in socio-behavioral studies of human generosity, the richness of stimuli via qualia producing architectures optimizes the organism's responsiveness (i.e., tunes and dilates) (Grant \& Dutton, 2012). Gamma signals in the human brain synchronize around the $30-90 \mathrm{~Hz}$ range, creating deeper absorption, imprint, and transmission capabilities in integrated information processing neural nets (Dobbs, 2005). Contemplation, or reflection as Merleau-Ponty prefers, is just one effect from consciousness's ability to either dampen or amplify systematic variances of qualia space from homeostasis. These variances physically resonate throughout the phenomenal background fields according to IIT studies and Keppler (Merleau-Ponty, 2014; Tononi, 2008; Keppler, 2016). These stochastic resonances, once integrated via SED, imprint both locally in classical physical forms, as well as non-locally in radiant quantum field encoding (Tononi, 2008).

Life in multidimensional, dynamic, variant states generates the contours of consciousness' ability to "play" within complex systems through endless speciation of equally endless combinations, structures, pathways, and its emergent interpretations of experience (Kak, Chopra, \& Kafatos, 2014). Narrating the transformational experience of a single atom named "X" in Odyssey, Aldo Leopold writes:

From his berth in the Indian's bones, $\mathrm{X}$ joined again in chase and flight, feast and famine, hope and fear. He felt these things as changes in the little chemical pushes and pulls that tug timelessly at every atom. When the Indian took his leave of the prairie, $\mathrm{X}$ moldered briefly underground, only to embark on a second trip through the bloodstream of the land (Leopold, 1949).

Speaking from a co-emergently reproductive and probabilistic perspective of biological recombinatory expressiveness throughout the vast scales of life's complex systems, Annie Dillard writes in Fecundity:

The faster death goes, the faster evolution goes. If an aphid lays a million eggs, several might survive. Now, my right hand, in all its human cunning, could not make one aphid in a thousand years. But these aphid eggs-which run less than a dime a dozen, which run absolutely free-can make aphids as effortlessly as the sea makes waves. Wonderful things, wasted. It's a wretched system. Arthur Stanley Eddington, the British physicist and astronomer who died in 1944, suggested that all of "Nature" could conceivably run on the same scheme. "If indeed she has no greater aim than to provide a home for her greatest experiment, Man, it would be just like her methods to scatter a million stars whereof one might haply achieve her purpose." I doubt very much that this is the aim, but it seems clear on all fronts that this is the method (Dillard, 1974). 
Perhaps then the big takeaway from Eco's poetics is his sense of how openness leads us away from the "Empirical Reductionist" stance by providing an interpretation of reality that is fundamentally "compositional" in its aesthetics. Paired with the emerging mechanics of quantum biological research from the rising tide of Hameroff, Penrose, Keppler, Lanza, and Laszlo, their empirical meeting ground appears to be the common sense of the phenomenal field dynamics at play. In the multidimensional, coherent ocean of experience, we can focus our attention to "fish" the waves of awareness, and infinitely catch ourselves into the subtlest depths of possible form. On the cosmic hook, which Hameroff refers to neuro-physiologically as the BING of quantum collapse-we plant our own bait amid a tide of SED oscillations in dynamic correlation, or resonance, to all fields of perception including ZPF (Laszlo, 2007; Edwards, 2017; Benzi, Sutera, \& Vulpiani, 1981). Some phenomenological feedback effects of such a system could be described as gratitude, emptiness, attunement, vibrancy, saturation, exhaustion, awake, and many other qualia forms. Yet in order to understand truth in our experiences, such as the qualia of "emptiness," according to CST, a conscious system must be fundamentally open or receptive to the state's subjective meaning, feeling, or frequency.

\section{Sources of Nature (How Does Expanding Awareness Affect the Mechanics, Dynamics, and Aesthetics of Conscious Experience?)}

In consciousness' quest to understand itself and its relationship to its context (in the universe), its role can be considered an integrative force within the equation:

$$
P^{?} \Phi / \int\left(U_{\text {corr }} \nabla R\right)=\forall|\aleph|
$$

where $P^{?}$ is an expression of pure possibility, $\Phi$ is the rate of conscious integration, $U$ expresses the totality of the universe, and $R$ is the stochastic field resonator. In this equation it is the correlation between the mechanics of possibility and stochastic resonance that allow openness to permeate many aspects of biological systems as experience's means of "quantum leaping" corporal discontinuities, such as attention, sleep, aging, and perhaps even death. A fundamentally open conscious system can potentially transcend "ship in a bottle" challenges articulated brilliantly by David Chalmers as, "phenomenal bonding or quantum holism (to solve the subject combination problem), small qualitative palettes (to address the quality combination problem), principles of informational composition (to address the structure combination problem), and a somewhat deflationary account of awareness of qualities to tie all these aspects together" (Chalmers, 2013; England, 2013). By treating such phenomena as compositionally integrated systems (with adaptive access to an entangled awareness described in ancient India as "Akasha"), which identify as individuals by correlative quantum mechanical means of embedded self-awareness and communication, Eco's "poetics of open work," emerges as a frame for the quantum biological interpretation 
of conscious experience (i.e., a semiotics of "being alive") on the basis of his representation of compositional works of art as aesthetic information vehicles, and coherent, open systems organized by consciousness for perceptual transmission and recombination into innovative forms (Eco, 1989). Eco's poetics include aspects of interpretability, communicability, sensitivity, formativity, and composition, which comprise the aesthetic basis for my MDA integrated systems approach.

Consciousness, in order to process its own significance, has evolved with life as its instrument to orchestrate elements such as light, water, earth, and air into ever more complex energy processing, critical information organisms. As biologist Jeremy England said in a 2014 interview, "You start with a random clump of atoms, and if you shine light on it for long enough, it should not be so surprising that you get a plant" (Wolchover, 2014). Correlatively speaking, consciousness can regenerate the very possibilities it consumes into greater possibilities, as established at least since the ancient philosophies of Aristotle and Buddhism, and later Anglicized in Transcendentalist writings of Emerson by such claims as "The sources of nature are in our own minds," and "It [consciousness] contradicts all experience." "How?" is a matter of transactional field dynamics, which communicate, organize and amplify resonance (i.e., information carrying signals) and radiance (i.e., energetic carrying signals) patterns throughout conscious systems.

Open systems can utilize patterns of attractors to tune Attentional capacities (a) into the interplay between Awareness $(A)$ and Probability $(P r)$, and in doing so loop in, constitute, and/or absorb the correlative nature of adjacent Possibilities $\left(P^{\prime}\right)$, ranging beyond its total radiance into unknown spaces of "no collapse." This is a notion constituted from John Wheeler's late assertion that, "the universe is filled with huge clouds of uncertainty, that have not yet interacted with a conscious observer or even some lump of inanimate matter. In all these places the cosmos is a vast arena containing realms where the past is not yet the past" (Lanza \& Berman, 2010). Thus an open mind can transcend itself by cohering various modes of perception, which as Lanza explains, "in terms of biology, the brain turns electrochemical impulses from our five senses into an order, into a sequence, into a face, into a room, into an environment" (Lanza \& Berman, 2010). The co-relative nature of observation is co-creative, and co-generative as well; and "Everything we observe is the direct interaction of energy and mind. Anything that we do not observe directly exists only as potential or mathematically speaking_as a haze of probability" (Lanza \& Berman, 2010). To Lanza consciousness also resolves into an integrated holograph, always in interdependent movement with its trends, or tides-dependent on how much harmonic force-as to prolong the dynamics of a universal projection (U). Lanza paints the picture further by reminding that, "Dreams and schizophrenia attest to the mind's compositional abilities to construct convincing realities," as well as, "The mind's awareness is the ultimate reality-paramount and limitless" (Lanza \& 
Berman, 2010).

As trends in attitudes toward Probability come and go, or aggregations of Awareness move in and throughout pockets of the projection, and as qualia burst through life, we can further witness and assess the emergent nature of "no-thingness" as much as thingness, space-time as much as collapse, gaps in attention with subconscious undercurrents of awareness. Through the phenomenon of the open mind the mirrors of our material existence become apparent, as does the understanding that observable matters do not inhabit much of the energetic landscape. One is reminded of the Zen saying, "Name the colors, blind the eyes," which underscores the effect of self-awareness arising from any experience. No longer can the perceiver merely make observations from outside, nor passively absorb experience. Thus, a chance encounter, such as "receiving intergenerational knowledge" contained in literary, pedagogical, and ontological vehicles-for instance reading about Pythagoras' Theorem-might engender a new sense of truth and interdependence in one's reality with causal implications upon local and non-locally entangled spin networks, as well as popular cultural ontology. Conscious coherence both evokes and exerts force (Eco, 1989). When a proof, song, or painting has the agency to become emotionally moving and phenomenologically relevant, even 2,000 years after its creation, the perceiver now participates in expanding the total field of conscious awareness, or the spirit of the work into new Possibility. Eco remarks, "To appreciate a work as a perceptible form means to react to the physical stimuli of the object, not just intellectually but also-so to speak-physically. Fraught with a variety of responses, our appreciation of the work will never assume the univocal exactitude and characteristic of intellectual understanding and will be at once personal, changeable, and open" (Eco, 1989). When experience imprints upon perceiver via their capacity for integrating Attention and Awareness, the interpretive process effectively extends the Awareness of composer, composition, and perceiver in Probabilistic and Possibility functions of universe (U). The phenomenal fields of perception therefore enact, channel, or play out constitutional movements of aesthetic information via the quantum mechanics of SR, as they ripple through the sensorial fields.

While materialist systems might contend that Beethoven's 9th symphony was composed in three or four dimensions, CST recognizes that the force of consciousness amplifies the musical score's integrative properties into quale that imprint the listener in perhaps sixteen dimensions, or more, of dynamic sensory information (Tononi, 2008). The effect of the performed music is transcendent of its material perspective via its ability to awaken higher order energetic and resonant senses in its audience, who absorb its aesthetic information in probabilistic realms beyond mere local embodiment-where the music can be heard-venturing into vast, subtler interplays of cultural, epigenetic, and subconscious Awareness entanglements-and therefore richer phenomenal potentials. 


\section{Information Processing Dynamics and Syntropic Organization in Biological Systems}

Hameroff and Penrose's quantum biological model of orchestrated consciousness via superposition events reveals a vulnerability within the standard model's negation of internal radiation factors when considering fluid mechanics within the differential equation of energy, and internal resonance factors within field dynamics of aesthetic information processes (Hameroff, 2014).

Eco cautiously considers that, "few people are willing to speak of meaning in relation to the kind of communication provided by a non-figurative pictorial sign or a constellation of sounds. This kind of openness is therefore best defined as an increase in information" (Eco, 1989). Yet, if in conscious systems, which are theoretically open (i.e., receptive), the energy coefficient of thermal conductivity, $k$, must consider

$$
q=k \nabla T
$$

where viscous dissipation function $\varphi$ may be positive due to properties of quantum processing, which augment the velocity field $V^{\star}\left(\nabla^{\star} T_{O}\right)+\varphi$ to $V^{\star}\left(\nabla^{\star} T_{O}\right)$ $-\varphi$, thus opening Newton's 2nd Law to reversible, albeit ultimately ephemeral, entropic flow processes when accounting for syntropy (King's supercausality) of internal radiation factors (White, 1999; Laszlo, 2007; Vannini, 2009).

In terms of IIT and OR correlative information processing effects (IPE), the standard model, which relies on elemental Cartesian controls, cannot account for the phenomenon of correlative field coherence (i.e., the quantum leap effect) in cognitive structures of living organisms. Phenomena such as language, bird song, neurogenesis in psychedelic interactions, neuroplasticity, epileptic seizures, musical scales, flight patterns of bees, and pattern encoding in water, transmit info via nonlocal fields and entanglement pathways to aggregate harmonic organization of internal resonance patterns into higher order behaviors through SED field dynamics (Keppler, 2016). IPE such as feelings, thoughts, epigenetics, memory, and dreams composite experience into forms of perceivable matter resistant to empirical methodology due to their irreducible internal qualia (q!) formations.

It can be said that biological systems that absorb, integrate, and transmit information via quantum processing architectures (i.e., microtubules) may also defy classical processes of velocity, acceleration, impulse, and entropy through conscious interpretive access to correlative fields of possibility ( $\left.\mathrm{P}^{3}\right)$, probability $(\operatorname{Pr})$, awareness (A'), attention (a), and experience (q!) where rate of processing $(\varphi)$ maintains an integrated coherence rate (i.e. homeostasis) of both radiance and resonance between all systematic dynamics $(\mathrm{Q})$. Keppler predicts that this correlation can be tested by measuring "the photon intensity and the theta rhythm," which theoretically aligns with Anirban Bandyopadhyay's research into orders of synchronization. Bandyopadhyay posits that every resonance in a conscious system creates a mental "singularity phase' with both a classical and quantum component. Yet spinning and nested within points of observation, re- 
sonant vibrations coherently transmit throughout the phase space's orders. Like Hameroff, he points to the role of tubulin proteins "dancing" within the microtubule resonance cavities as fractal resonance chains, that connect the biological "clocks" of conscious systems, while allowing for multivariate responsiveness based on cycles of geometrically natural number systems (Bandyopadhyay, 2016).

Overlapping phase stimulation triggering neural spikes allows axons to translate resonance into integrated composite formations from the interplay of field dynamics within the particular receptivity of different conscious systems. These qualia compositions and their IPE can be expressed in terms of harmonic behavioral dimensions and interactions of their fields correlative to $\varphi$ and ZPF (Benzi, Sutera, \& Vulpiani, 1981; Kanai, Minemoto, \& Sakai, 2005). Whereas, "For a machine there is no other principle but physics-unlike a man or a pigeon they do not have the unitary sense experience necessary for perception and self-awareness" (Lanza \& Berman, 2010).

Case study of syntropic compositional effects of various systematic orders might include: people who can moderate body temperature, variance of emotional impact of an audience watching a film, evolution of interspecies communication or cooperation, water's role in thermodynamic regulation of quantum biological processes, sensory deprivation and neuroplasticity adaptation (i.e. hypoxic training techniques in mountain climbers), mental health benefits of exposure to non-tempered music forms, evolution of migratory patterns and/or diet and/or reproductive habits in animals, mycelium connectivity functions in forests, and the morphological evolution of "top-down" visual processing in mammals and humans (Kozhevnikov et al., 2013).

Entropy, however, provides an important and inevitable balance-a cosmic tide, or push to consciousness' pull-correlative to its total perceivable radiance, which is aggregated by Awareness. For instance, most people believe the likelihood of extraterrestrial life in the universe, and our mathematical probabilities encourage this belief. So it may be that if we currently find no other evidence of life in the universe but our own planet's, it isn't so much that it fails to exist, rather, we might agree that our local consciousness hasn't yet learned to recognize itself in certain non-localized forms. One precedent exists in cultural studies of an evolutionarily emergent linguistic awareness of the color "blue" in some ancient societies. ${ }^{1}$ Much possibility for undiscovered extraterrestrial life is left open, since due to entropic discontinuities, or interference, it could be aware, or not aware, of us as well. That's the balance.

As Rumi describes, "Consciousness sleeps in minerals, dreams in plants, wakes up in animals, and becomes self-aware in humans" (att. Rumi). It is in consciousness' awakening (i.e., dynamic self-awareness) that we critically formulate and enfold our relations to all of creation, even as all of creation unfolds. In everyday life, choices are narrowed down to specific possibilities. Superposi${ }^{1}$ If it's true, as posited by philologist Lazarus Geiger, that Egyptians were the first society to develop awareness and a word for the color blue. 
tion is routine. It is the same phenomenon that leads Aristotle to proclaim, "To be conscious that we are perceiving is to be conscious of one's own existence," even as Emerson cautions in The Over-Soul,

The influence of the senses has, in most men, overpowered the mind to that degree, that the walls of space and time have come to look solid, real and insurmountable; and to speak with levity of these limits is, in the world, the sign of insanity. Yet time and space are but inverse measures of the force of the soul. A man is capable of abolishing them both (Emerson, 2004).

Social habits of Attention and Awareness, as studied in ethical philosophies, therefore have a great impact on what's perceptively true at any given time or place in our existence. Lanza points out that, "Until Jules Verne and others wrote about humans going to the moon in the $19^{\text {th }}$ Century, it was too fantastic a notion to spread widely. By the 1960s however man's space travel had become such a common sci-fi theme that it was an easy sell to the public, who readily agreed to fork over taxpayer dollars to turn it into a reality during the Kennedy, Johnson, and Nixon administrations" (Lanza \& Berman, 2010). It's a dynamic of the evolutionary indeterminacy of perception, discussed by both Lanza and Eco, that transcends the random physical notions of mere "relativity" - in play as Einsteinian principles are (Eco, 1989). The quantum layers of our existence therefore are not just relative, but also "co-relative to mechanics of consciousness' criticality in perception and cognitive interpretation. Instead of being ontologically hemmed in at observation, consciousness, and its chariot of embodied life, can transmit crystalline holographic projections of experience in poly-dimensional resolution. Eco calls on the writings of Sébag commenting on Marx:

As Marx writes, consciousness is not only the consciousness of a reality outside itself but also its own being. This does not mean the subject is immediately and intuitively present to itself, but rather implies a system of laws that are not imitated but rather are acquired from and through the progressive use of an intelligence that is coming to grips with a universe of objects. These laws can in turn be transformed into instruments, since the organization of reality, as well as the discovery of the order that underlies it, depends entirely on them; on the other hand, this reality is none other than the very source out of which the intellect draws the meaning of its own logical organization (Eco, 1989).

Consciousness's responsive ability to evaluate feedback within the body's instrumental resonance chamber, or to cry at a film's tragic ending, shines light on the resonant quantum biological dimensions of transcendence. Examples include resonant schema within the semiotic composition of various folk ontological systems from acupuncture to yogic astral planes to Leary's "8-Circuit Intelligence." These culturally emergent systems organize and articulate subtleties of 
existence co-relatively mirrored in the dynamics of butterfly effects, the structures of quasars, or democratic elections (Mitchell, 2013). Regardless of form, as the force of consciousness appears throughout our perceptions, "staying open" to the compositional possibilities of new forms augments systemic dynamics and optimizes critical communications operants. As Eco says,

How often have new creative modes changed the meaning of form, people's aesthetic expectations, and the very way in which humans perceive reality? The poetics of the open work is an expression of such a historical possibility: here is a culture that, confronting the universe of perceivable forms and interpretive operations, allows for the complementarity of different studies and different solutions; here is a culture that upholds the value of discontinuity against that of a more conventional continuity; here is a culture that allows for different methods of research not because they may come up with identical results but because they contradict and complement each other in a dialectic opposition that will generate new perspectives and a greater quantity of information.

After all, the crisis of contemporary bourgeois civilization is partly due to the fact that the average man has been unable to elude the systems of assumptions that are imposed on him from the outside, and to the fact that he has not formed himself through a direct exploration of reality (Eco, 1989).

When every part of existence is playing its part, special relativity is only the most dynamic, observable, physical aspect of a materialistic epistemology. By including the phenomenological realms of perception formulated by aesthetic information, "The distance between a plurality of formal worlds and undifferentiated chaos, totally devoid of all possibility of aesthetic pleasure, is minimal" (Eco, 1989). Interpreting what is felt as much as what is quantified during conscious integrative processing, the poetics of experience becomes richer by magnitudes, and much more attuned to the wonders long-articulated in spiritual traditions and art. The explanation for all the processing power in our guts suddenly makes a lot more sense too; not as a matter relative to "what you are," so much as co-relative to "how open you are" cognitively during imprinting of conscious experiences. The co-relative factor of a perceiver's "openness" as they integrate experience into co-reality, affects the quality of the information they receive and transmit. There is also a self-regulatory aspect to the feedback of such systems that favors openness in modes of survival. Eventually openness provides greater agency of will than predominantly "closed" systems. Concentrated or narrowed systems, or dissonant systems, will facilitate fewer orders of aesthetic complexity when integrating an evolving spectrum (i.e., dynamic, at criticality) of conscious information, since they are less adept at accommodating a comparable bandwidth for processing qualia and IPE. Confronting the artistic exercise of will sparks Eco into more radical modes. "The moment an artist realizes that the system of communication at his disposal is extraneous to the his- 
torical situation he wants to depict," Eco writes, "he must understand that the only way he will be able to solve his problem is through the invention of new formal structures that will embody the situation and become its model' (Eco, 1989).

He's speaking to a communicative factor that might correlatively view mycelium as more effective communicators for a thriving forest, than perhaps a corrupt government for its oppressed constituents. The artist's ability to approach their own fulfillment with an open mind is Eco's key to an awareness which transcends material limitations, locio-temporalized physical existence, and other improbable discontinuities in order to sense connective possibilities in all experience, while simultaneously sculpting new forms from life's choices as they arise. When getting one's way and not getting one's way are consciously, equivocally interesting outcomes on the same Maslowian "plateau," consciousness opens critical cognitive functions to new integration imprints and composition of higher ordered experience. Skillful interpreters can thereby innovate narrative structures which update understanding and promote internal radiance factors (i.e., syntropic processes) non-locally.

Simultaneously, at any given point in our conscious experience as humans, we are choosing where to place our attention, and how to respond to the relationship (possibilities, probabilities, and feedback from cultural awareness) it generates with its attractor. Is a sound noise or music? A video chat may be more convenient and a more pleasurable alternative than "never seeing your child when traveling on business," but the transmission of your child's presence-their visage, their weight, their smell and feel, their total field presence (TFP) of radiance and resonance factors-cannot be substituted via mediated experience. In other words, there's no "app for that." For instance, in "digital," pathways for the resonant factors of touch as a communications medium are at least reduced and at most unconvincing. If TFP constitutes the entire radiant spectrum of an organism's aesthetic information transmissions, the system's conscious integration rate, or coherence can be expressed at $\varphi$ where:

$$
P^{?} \varphi / U \geq 1
$$

An organism's ability to remain in coherent attentive states affects $\int\left(U_{\text {corr }} \nabla R\right)$ in terms of total radiance. If reflection forces us to choose and focus on just a few attractors of a given situation, "the undefined pervasive quality of an experience is that which binds together all the defined elements, the objects of which we are focally aware, making them a whole" (Eco, 1989). When inevitable trends of decoherence occur during processing variations, radiation in total terms of $U$ wavefunction $(\psi)$ persists during all phases of consciousness. Thereby, an event such as death can have varying circles of supercausal impact dependent upon the superposition gap in total generative radiance and resonance of $U$. Such a phenomenon suggests one way to understand the impact of a public figure's passing in relation to the intimacy of someone passing from within more private 
spheres. Nonetheless, all systems eventually lose coherence-even David Bowie, stars, quasars, and memes. All systems eventually fail in application: open, closed, radiant, irradiant, organic, synthesized, or transcendent. One day the projection of the universe will likely go dark.

For now, one's Attention has become a precious human resource (just look at contemporary advertising strategies, given to historical compositional factors that "affect attitude formation and circumstances that enhance or diminish the need for cognitive closure [that] can affect thought processes and outcomes in ways that mimic dispositional openness" (MacKenzie, 1986). Readily manipulated conditions, "such as ambient noise, threats of physical violence, danger, paranoia, dullness of task, or imposed time limits, can make individuals functionally closed" (McCrae, 1996). Clearly Attention plays a significant role as the gateway to the liberation or dissolution of the richness of conscious experience, whose bandwidth greatly fragments within conditions such as highly mediated interfaces and the cultural scenarios dreamed by Kerzweil and other Transhumanists (Schneider, 2008; Kaufman, Quilty, Grazioplene, Hirsh, Gray, Peterson, \& DeYoung, 2015). As the eyes have evolved to become humans' primary directors of information gathering, "likely due an evolutionary transition from being nocturnal, arboreal and relatively solitary, to being diurnal, ground-based and social," the harnessing of that direction into ever more galleries of digital absorption drives even more co-opting of our total sensory attentiveness into concentrated sensory pathways, which potentially condenses $P ?(\varphi)$ by limiting the dynamic imprinting of SED harmonics during aesthetic absorption; effectively causing some attentional preferences to under develop, while others exhaust overstimulated pathways, and therefore cause habituation of underutilized spectrums among other sensory pathways (Passingham \& Wise, 2012).

For instance, the colonialist hegemony in Western attitudes towards "touch" between two humans, has largely minimized openness to touch, as well as social awareness or consciousness of the possibilities of dynamics in "how we touch" or why (Butler, Laclau, \& Žižek, 2000). Yet it is clear that "Being touched by another person influences our readiness to empathize with and support that person." In a 2011 study, "touch enhanced event-related potential (ERP) correlates of picture processing. Pictures elicited a larger posterior N100 and a late positivity discriminated more strongly between pictures of neutral and negative content when participants were touched" (Schirmer et al., 2011). The source of the prevalent contemporary semiotic dynamics of "touch" can be traced to influential Puritan and Victorian morality systems whose communicability through the hegemonic influences of Colonialism, has led to current social constraints (regardless of ethical stances within the topic) trending towards the disapproval of being touched by "a stranger," or preferring being touched by a "lover" to another individual. There are well known morphological implications written into cortical structures and neurological behavior that are major evolutionary contributors to these preferences and their conscious transmission as 
"attractors" of attention² (Raju, 1980; Eccles, 2005; Vannini, 2009; Mattson, 2014). Furthermore, research into the cognitive ethology of "vigilance or scanning behavior in highly social birds" also reveals related dynamics in the philosophy of mind in processes of gathering information and representation" (Bekoff, 1995).

In general, the more open we are to our experience via the interplay of our aesthetic information absorbing attention, as it accesses quantum events from dynamics in fields of awareness, probabilities and possibility, the more richly we experience the mechanically attuned fine structures of our consciousness radiating or emanating in its many phases and forms throughout the universe. In her book, The Quantum Self, Dana Zohar claims, "Our minds are interwoven with memory. Our bodies-apart from skills-are blind to all but the moment" (Zohar \& Marshall, 1990). I would argue this claim is false, and that our bodies are as ancient as "the moment." Do we not contain stardust from the big bang itself? Don't we still live and perceive even the slightest mundanity embedded within a CMB dependent reality (Kohri, Lin, \& Matsuda, 2014)?

Or consider how Eco puts the moment of experience and attention in terms of works of art:

Now it [the open work] appears as the concrete solution of the "quarrel" between the "question of poetics" (here understood as a formal model which has been and can be elaborated within the context of a cultural discourse, and which need not assume the form of a concrete artistic object) and a "physical organism" (which in numerous cases is really only a temporary and inessential vehicle for the ingenious solution of a question of poetics).

And this is precisely why those works that investigate contemporary poetics have a validity that takes precedence over other critical processes: they make room for choice-provided this choice is not expected from the theoretical investigations of aesthetics, on which the very conditions of the choice rest, or from the investigations of cultural history, which are mostly concerned with the historical developments of both poetics and criteria of choice (Eco, 1989).

In scientific exploration for a "criteria of choice," Penrose turns to cricket balls to suggest the evolution of our theoretical views:

Cricket balls are indeed well approximated by the descriptions of classical physics. They have reasonably well-defined locations, and are not seen to be in two places at once, as the linear laws of quantum mechanics would allow

\footnotetext{
${ }^{2}$ Vannini deftly covers morphological attractors in cognition by stating: "This model implies that all the components, from the molecular one to the global brain structures, can reciprocally activate each other. The fractal nature of their connections, the sophistication of neurons and synaptic junctions, leads to a modular and flexible structure. According to King, the anticipatory properties of these systems, their flexibility and ability of performing decisions, justifies why this model has been selected during evolution. The advantage of conscious processes in terms of anticipation, flexibility, learning and self-organization are fundamental for the survival of the living system and therefore free will and consciousness have emerged, surpassing any eventual computational systems."
} 
them to be. If the procedures $\mathrm{U}$ and $\mathrm{R}$ are to be replaced by a more comprehensive law, then, unlike Schrodinger's equation, this new law would have to be nonlinear in character (because $\mathrm{R}$ itself acts non-linearly). Some people object to this, quite rightly pointing out that much of the profound mathematical elegance of standard quantum theory results from its linearity. However, I feel that it would be surprising if quantum theory were not to undergo some fundamental change in the future-to something for which this linearity would be only an approximation. There are certainly precedents for this kind of change. Newton's elegant and powerful theory of universal gravitation owed much to the fact that the forces of the theory add up in a linear way. Yet, with Einstein's general relativity, this linearity was seen to be only an (albeit excellent) approximation-and the elegance of Einstein's theory exceeds even that of Newton's (Penrose, 1999)!

It may be that Penrose's openness evokes a theoretical shift into a paradigm of "leaky" poetics that in some respects "chooses us." Also, Eco's perspective compounds the potential for theoretical adaption within OR Theory's scenarios by correlating qualia in terms of receptivity to IIT's expanded dimensionality of absorption, integration, and transmission. Eco's "criteria" factors into the perceptual that, "Like Proust's or Whitehead's or Einstein's world, “Joyce's world is always changing as it is perceived by different observers and by them at different times," bringing an important twist to Penrose's helical modeling by identifying that "...contemporary poetics rebels against the psychic inertia that has been hiding behind the idea of a recovered order" (Wilson, 1961; Eco, 1989).

\section{Perception's Bridge (What Are the Neural Correlates of an "Open Mind"?)}

Consciousness is having an experience. Due to biologically observable architectures within qualia producing systems, such as microtubules in the human brain, photosynthesis in plants, and information patterns in water and light; the perceivable existence and nature of living systems of radiant energy within the ZPF, creates within our universe qualitative coherence in terms of SED resonance and the anthropic principles, which allows sensory processes to imprint information $\left[\mathrm{P}^{?} \varphi\right.$ from $\mathrm{Q}$ states] between nonlocal, indeterminate, and discontinuous fields via responsiveness to multidimensional dynamics integrated into organically receptive, compositional aesthetic patterns (such as synchronization, mimesis, poesis, and projection), which contribute increasingly complex, rich, orchestrated, and open transmissions, or effects, of phenomenal experience to the total radiant projection's force, experience, and presence throughout the system.

In humans, in order to "bridge" the psycho-physical restrictions of animal perception, consciousness acts as an integrative force between somatic activities including cortical and basic laws of the universe, which are both discontinuous and indeterminately dependent on SED state oscillations and range coherence. Qualia result as highly organized mental forms with "actual" multidimensional 
capabilities for synthesizing and storing raw aesthetic components in richly orchestrated patterns of information such as feelings, emotions, thoughts, memories, and habits.

An open mind gains amplification via the senses, biofeedback (i.e., thought), directionality (i.e., spin), and through both local and nonlocal systematic resonances (e.g., gravitational) - thus arriving at a geometrical processing aesthetics proportionate to Tononi's experimental 16-D qualia shapes as illustrated below in Figure 2 (Nystrom, 2004; Tononi, 2008; Sarkar \& Bhattacharyay, 2014).

The orders of probabilistic experience give rise to Sébag's "progressive use of intelligence," which allows experience to generate "instruments" or "technologies" of perception from recursive qualia interpretation. Eco adds to the critique of formativity, "The moment consciousness recognizes the object, it gets rid of its alienation by negating the object itself" (Eco, 1989). However, in light of Keppler's research, the object and observer are both imprinted with information depending on their SR interface architecture and adaptive openness. These transactional amplification processes, when represented in frequency-specific,

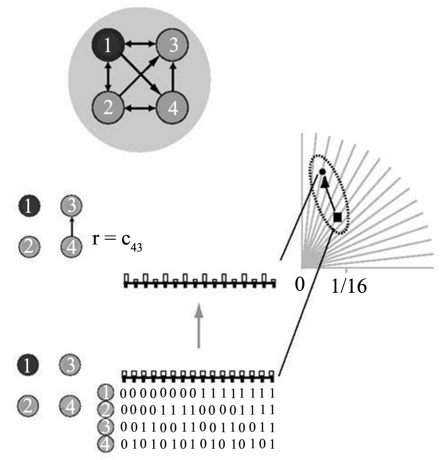

(a)

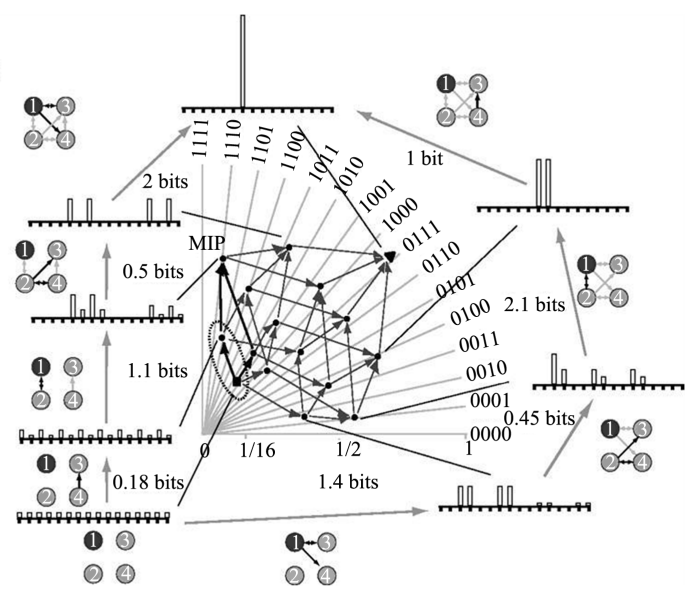

(b)

Figure 2. IIT's qualia geometry. As information states arise via qualia production (i.e., integrative information compositional events from background stimulus), aperiodic resonance structures network and crystallize (i.e., imprint) through phase shifts. Qualia (q!)-space for a system of four units is 16-dimensional in its variant states. The figure shows 16 out of the 399 points in the quale, generated by combinations of the four sets of connections. The probability distributions depicted around the quale are representative of the repertoires generated by two q-edges formed by q-arrows that engage the four sets of connections in two different orders (the two representative q-edges start at bottom left-one goes clockwise, the other counter-clockwise; black connections represent those whose contribution is being evaluated; gray connections those whose contribution has already been considered and which provides the context on top of which the q-arrow generated by a black connection begins). Repertoires corresponding to certain points of the quale are shown alongside, as in previous figures. Effective information values (in bits) of the q-arrows in the two q-edges are shown alongside. Together, the q-edges enclose a shape, the quale, which completely specifies the quality of the experience (Tononi, 2008. Consciousness as integrated information: a provisional manifesto. The Biological Bulletin, 215(3), 216-242.). 
harmonic wave patterns, as found in the 2016 study Exploring Neural Correlates of Consciousness with Connectome-specific Harmonic Waves (Atasoy et al., 2016) shows that:

Spatial correlation patterns of the resting state networks (RSNs) are predicted by the harmonic standing waves; i.e. resonance patterns, emerging on the human connectome. These harmonic waves, estimated by extending the Fourier basis to the particular topology of the human connectome, provide a new analytical language for cortical activity. In this new frequency-specific representation, RSNs significantly match harmonic wave patterns of certain frequencies. A neural field model of excitatory-inhibitory neural activity provides a biologically plausible neural mechanism behind the self-organization of these resonance patterns. Remarkably, the critical relation between the simulated patterns and the delicate excitation-inhibition balance fits the neurophysiological changes during local cortical dynamics.

These findings demonstrate that the fundamental principle underlying resonance, ubiquitous in nature (e.g. acoustics, electro-magnetic interactions, electron orbits and morphogenesis), likely underlies macro-scale cortical dynamics and provides a new tool to investigate the neural mechanisms underlying local cortical dynamics (Atasoy, Donnelly, \& Pearson, 2016).

Furthermore, new mathematical research by Anirban Bandyopadhyay adds, "We have determined the mathematical origin of distribution of resonance frequencies, and it appears that nature relies on the number system [i.e., Platonic, non-algorithmic] to create materials and composition of frequencies" (Bandyopadhyay, 2016).

In the case of neural resonance patterns in the practice of meditation, a breathing practice which correlates to increased gamma synchrony as well as "extended prosociality and tolerance of outgroups, at least among those with socio-cognitive and moral openness;" "staying open" to the raw neural impedance of emotions and impulses that arise when a novice begins practice, as opposed to the highly resonant equanimity and calm of a seasoned practitioner, relates to the differentiation of NCC results between the two practitioners' "cognitive bandwidth" for their individually "tuned" systems' bio feedback across connectome specific neural patterns (Lutz et al., 2013; Clobert, Saroglou, \& Hwang, 2015). The novice may experience greater fluctuations in comfort, while the expert can withstand a more dilated, "plateaued" and deeply pleasurable absorption of informational dynamics in contrast to homeostasis.

Similarly, Walter J. Freeman, reveals that NCC were found in the ECoG of animals and in the EEG of humans after training to discriminate conditioned stimuli (CSs). Importantly, stimulation occurred in each action-perception cycle $(\mathrm{a}-\mathrm{p})$ as amplified rates of "meaning," not "representation." The study's abstract also notes, "Brains being open thermodynamic systems, a complete description must include the environmental sources, whence come life-sustaining 
matter and energy, and the environmental sinks for wastes, heat and entropy" (Freeman, 2016). Even in generating the degrees of freedom that can mirror predictive environmental modeling, it is still vital to "acknowledge the presence of the permanent mental structures by virtue of which "the physical world reveals the organization that transcends it by abandoning itself to our perceptions" (Eco, 1989). For instance, new brain imaging studies with psychedelics makes a case that underlying the psychedelic-state induced by these drugs is an increase in the level of entropy of cortical activity, i.e. cortical activity becomes less predictable or more disordered (Carhart-Harris, 2016). Yet follow-up testing for falsifiable increases in order-due to latent neurogenesis in cortical responsiveness-may reveal results subversive to classical "laws." Overall it is heartening to discover the field of consciousness' readiness to push out of Newtonian limitations from respected sources such as Stuart Kauffman, who relates, "With Newton we lost our minds and became disenchanted. Newton, in classical physics, gives us a view of the world as an entirely entailed unfolding. Nothing not entailed can happen. Due to this causal closure of classical physics, a classical brain can at most witness the world, not alter it, so be at most epiphenomenal. Then why have we evolved such complex brains? The best hope for a more-than-epiphenomenal mind requires quantum mechanics, QM. "Choice" implies that we could, counterfactually, have chosen otherwise. This is ontologically possible in QM if measurement is real and ontologically indeterminate" (Kauffman, 2016).

\section{Compositional Responsiveness in Biological Systems}

Umberto Eco's poetics of openness matches with Stuart Hameroff and Roger Penrose's OR Theory in terms of both works' interpretation of quantum mechanics within systems capable of dynamically integrating aesthetic information.

"Staying open" is a biological phenomenon of responsiveness to conscious information integration, according to qualia producing processes described by Tononi in IIT, which is to say, that the quality of information interpretation in a living organism depends on its responsiveness to its perceived compositional possibilities. In theatre school we used to have the saying, "How a character breathes is how a character thinks." Similarly, how an organism composes and engages its energy with the sensations and information from didactic or ambiguous stimulus is often correlated to how it feels in relation to the stimuli. Therefore, perceptions live and thrive in a resonant, coherent projection of harmonic radiance, with an array of integrative mechanisms, field dynamics, and phenomenological aesthetics. In recent work, Katherine Peil (2016) explains:

When phenomenal experience is examined through the lens of physics, several conundrums come to light including: Specificity of mind-body interactions, feelings of free will in a deterministic universe, and the relativity of subjective perception. The new biology of "emotion" can shed direct light upon these issues, via a broadened categorical definition that includes both affective feelings and their coupled (yet often subconscious) hedonic 
motivations. In this new view, evaluative (good/bad) feelings that trigger approach/avoid behaviors emerged with life itself, a crude stimulus-response information loop between organism and its environment, a semiotic signaling system embodying the first crude form of "mind". Emotion serves the ancient function of sensory-motor self-regulation and affords organisms - at every level of complexity - an active, adaptive, role in evolution. A careful examination of the biophysics involved in emotional "self-regulatory" signaling, however, acknowledges constituents that are incompatible with classical physics. This requires a further investigation of the fundamental nature of "the self" as the subjective observer central to the measurement process in quantum mechanics, and ultimately as an active, unified, self-awareness with a centrally creative role in "self-organizing" processes and physical forces of the classical world. In this deeper investigation, a new phenomenological dualism is proposed: The flow of complex human experience is instantiated by both a classically embodied mind and a deeper form of quantum consciousness that is inherent in the universe itself, implying much deeper-more Whiteheadian-interpretations of the "self-regulatory" and "self-relevant" nature of emotional stimulus (Peil, 2016).

From the perspective of CST, the "new phenomenological dualism" Peil proposes resolves into a larger coherence found in Merleau-Ponty and Keppler's field dynamics, which Keppler designates within the ZPF, and I describe for popular mimetics as "the Outernet"-the phenomenological field harmonically connecting all perceptual and sensorial fields. Nevertheless, Peil clarifies how all living organisms are capable of basic orders of perception grounded in their ability to process information, and those of higher order processing capabilities amplify it through cognitive architectures such as memory and language. According to the autogenesis Terence Deacon ascribes to viruses and microtubules alike, perception can draw upon the morphodynamic powers of reciprocal catalysis and self-assembly to transcend boundary conditions, and "ratchet" into higher synergetic orders (albeit subject to decay and entropy) (Deacon, 2016). In higher orders, expressions of environmental mirroring, matching, attracting, pattern recognition, and play can transmit synthesized aesthetic information as expressions of possibility, choice, behavior, and art in humans.

Sound is an effective medium to track through the human nervous system. Albeit only a certain spectrum of frequencies is processed through the ears, its waves are absorbed throughout sensory resonators, and its information stimulates the body's choir of microtubules located within neurons throughout the system. It may be that water keeps quantum processing within the microtubules cool, and attunes its sensitivities to sound stimulation via interaction with hydration reservoirs, unless disruptive feedback from the organism, such as emotions or strenuous physical exertion, generate interference patterns (Vannini, 2009). The microtubules integrate sound vibrations into the qualia-shape compositions described by IIT's geometry. Experiential sequencing remains coherent 
unless interrupted by chemical disturbance as in the case of an epileptic seizure or disarmed pi resonance clouds in the case of anesthetics. Otherwise the organism responds in its own time, or kairos, (i.e., stays open) with its abilities to engage, create, communicate, act, and contemplate (i.e., respond) co-relatively to the sound vibrations it receives.

Sound, as a localized, yet indeterminate, basic phenomenon or possibility in biological experience on our planet, represents a source of aesthetic information common to every living organism no matter its processes for integration. Every organism, and even water, can respond to sound vibrations in some way, even if it's not through "hearing." The compositional properties of sound perturbations as studied in the field of cymatics are vast when amplified by the fields of perception, directionality, and the physical dimensions in correlation with ZPF (Holmes \& Danielson, 2012). The harmonic complexity and biological impact of sound's resonant properties' agency to compose aesthetic responsiveness within evolutionary organization gathers support from the research of both Katherine Peil and biologist András Balázs (Balázs, 2004; Peil, 2016). As organisms gather more sensitivity to the resonance factors of certain mediums, such as sound, the feedback generated within the organism allows for increased processing and higher orders of conscious behavior based on the organism's ability to openly interpret, rather than narrowly, the multidimensional compositional possibilities of the information it receives. By adapting to an open response-ability, rather than a co-relatively closed or merely reactive functionality, life effectively leverages its experiences to galvanize compositional possibilities via probabilistic pathways of awareness and attention into ever-greater complexities of organization, pattern recognition, creativity, and transmittable forms of conscious expression. "Staying open" to the Outernet becomes an important phenomenological aspect of consciousness' syntropic temporal agency to evolutionarily compose, access, understand, and innovate its existence, and offers the foundation for the essential arising of humanity's astounding musical achievements, as well as its universal propensity for appreciating the complex scorings of artists like Bach or Mozart (Belluck, 2011).

Orchestrated experience (OR Theory) in terms of receptivity to IIT's sixteen dimensions of absorption, integration, and transmission offers enrichments in the understanding of biological catalysts like sound that correlate to how living organisms learn to cooperate with increasing creativity, or openness to attractor outcomes, as well as toward the conceptual field of aural possibilities. Sound travels openly through these fields of proto-consciousness within biological systems within physical range of feeling or detection.

Therefore, biological systems, as formative, energetic, and information processing models in functional quantum coherence with anthropic ZPF principles, consciously convert aesthetic information (as feelings and other expressions of mind) into irreducibly synthesized compositions, like salt in classical physical terms, or qualia via the phenomenon of a field dynamics based on open inter- 
pretability. The openness, or receptivity to such qualia generating events in coherent, biological systems thereby commutes throughout conscious experience.

A notable physically adaptive correlation is the evolutionary phenomenon of running in mammals. Whether in the case of cheetah, deer, or human, running consciously increases mobility and spatio-temporal awareness by evolving predictors of location and predation that significantly complexifies cognitive pattern processing.

Among mammalian species there are significant positive correlations between brain size, cognitive abilities, and exercise capacity. Studies of rodents, monkeys, and humans have shown that running can increase the size of several different brain regions including the hippocampus and midbrain. Presumably, individuals whose brains responded to endurance exercise by increasing the growth of their brain cells would have a survival advantage because of the superior pattern processing ability conferred by the additional neural circuits. Indeed, at the cellular level running can increase numbers of synapses and the production of new neurons from progenitor cells in the hippocampus. The ability of running to improve pattern processing is evolutionarily conserved, as demonstrated in experiments with rats and mice showing that running enhances hippocampus-dependent spatial pattern separation. In humans, running improves mood and enhances cognitive and sensory-motor capabilities, and running also enhances cognitive performance in monkeys (Mattson, 2014).

As a sustained activity, running coordinates parallel cognitive architectures of proprioception, systemic homeostasis indicators (i.e., breath and heart-rate), control neuron pathways in attention, edge detection, and both top-down and bottom-up decision-making. The runner's ability to consciously regulate, perceive, or "relate" to, these co-emergent processes composes experiences ranging from intense survival scenarios to intense feelings of joy, depending on the subject's responsiveness to its environment and interpretation of internal perturbations correlative to equilibrium states. If the runner consciously opens, or "chooses" to prefer creative potential within their own pursuit, the probability of innovative behavior organizing its awareness temporarily compounds, as does attentional receptivity to innovative behavior, albeit moderated by attractor habituation patterns. For instance, an "open" runner is free to create his or her own route with varying dynamics of spontaneity, efficacy, play, didacticism, ambiguity, and experience potentials towards realizing their goal (i.e., associate with) in co-relatively speciated degrees, or aesthetics, of their consciousness' threshold for engaging the activity of running. Observation once again corroborates the predictive relevance of Hameroff's reasoning a "quantum pleasure principle," as it arises in mammals who can run: their young learn to appreciate the running early on through any action-perception cycles (a-p) of spontaneous "fun" biofeedback (CSs) produced from sustained, embodied, complex coordi- 
nation (Hameroff, 1999; Deacon, 2016; Freeman, 2016). In turn, running evolutionarily commutes/opens innovative complex pattern responsiveness, temporary increased order within the organism, greater processing capabilities to its conscious adapters, and other potentially epigenetic traits.

\section{The Bell of Truth (How Does Open Mindedness Reveal the Nature of Consciousness?)}

The non-algorithmic harmonic properties of conscious information processes provide a deeper understanding of how open, qualitative characteristics are biologically and evolutionarily, co-relatively preferred to closed, quantitative capacities alone. The ever-available phenomenon of "staying open," as a critical choice of will, may therefore lead to an argument regarding "why and how," "When we think and perceive there is a whir of information processing, but there is also a subjective aspect" (Chalmers, 1995; Lanza \& Berman, 2010). If dissonant information transmissions, like alienated musical chords devoid of compositional context, are both difficult to interpret and share, harmonious bundles of information can be described as optimal to maintaining higher frequencies of coherence, more interpretable wave patterns (i.e., resonance), scalable synchronization across orders, a wider stream of informational access points, more evolutionarily efficacious and communicable signals, and narrative enfolding possibilities for fewer space-time discontinuities. One might say: without a ring of truth, no bell could be heard, let alone ring.

In Aristotelian poetical systems, a character's fate is determined in terms of his cognizance of possibilities within the most tragically improbable circumstances (Donini, 2010). A great cultural example for mirroring harmonic narratives at play within a field dynamics of consciousness is Hamlet. As a work resonant with Greek and contemporary aesthetics, its main character suffers the poisoning of his noble lineage or cultural narrative which is thrown into dissonance by incestuous envy, therefore nullifying his entire political regime, and his paternal society's agency to regenerate a virtuous future. Hamlet's ability to openly perceive his fate, while simultaneously experiencing his inability to escape or separate himself from its inevitability, leads to the revelation of his character's articulate degrees of great truths, pursuit of good by his demise, and the beauty of his death-rightfully earning him the classical status of "great tragic hero." Hamlet tries twice, unsuccessfully, to exercise the agency of his dramatic consciousness by writing himself a new ending: once by enacting the play for Claudius, and secondly by switching notes on Rosencrantz and Guildenstern so that he might escape to England alive. However, since Shakespeare is the author and not Hamlet, and since Shakespeare has decided to tell a tragic story: Hamlet must die. One of drama's greatest moments is achieved by Shakespeare's allowing Hamlet to freely surrender to his dramatic fate (Shakespeare, 1904). In Act 5, Scene 2, aware of Laertes' plan to spar with a poisoned blade, Horatio offers Hamlet one more chance to escape: 


\section{HORATIO}

If your mind dislike anything, obey it. I will forestall

their repair hither and say you are not fit.

\section{HAMLET}

Not a whit. We defy augury. There's a special

providence in the fall of a sparrow. If it be now,

'tis not to come. If it be not to come, it will be now. If it

be not now, yet it will come-the readiness is all.

Since no man of aught he leaves knows, what is't

to leave betimes? Let be.

Furthermore, the appearance of the Ghost of Hamlet's father symbolizes an experience where Possibility and Awareness collaborate masterfully to manifest an improbable event. The ghost embodies how the court of Elsinore feels subconsciously about the King's recent death-he bears as much information in his dialogue and purpose-and so upon appearing before Hamlet, he says what everyone "already knows" subconsciously, yet in a form that can only be perceived by characters Shakespeare has written as "open to hearing the truth"-no matter how terrifying it may be. According to Heisenberg's uncertainty principle, the probability of seeing a ghost of your dead father rises significantly in terms of quantum resonance dynamics when there is a great possibility that you feel he died acrimoniously. Indeed, Hamlet must co-create the ghost so that he can cognize the truth latent in his own awareness. It is the Open Mind's ability to walk through imaginary doors and arrive in real predicaments that allows consciousness to weave together Possibility and Awareness by co-relatively transcending Probability. This is a process that evokes outcomes of entanglement destined to some degree of systemic failure after integration processes exhaust their local possible outcomes. Thus, although the Hamlet character must die, the Hamlet phenomenon lives immortally in our Awareness, precisely due to its resonant ability to "actually" integrate with an open mind.

In contrast to plays, another kind of technological system, the Internet, might be considered a magic mirror that can virtually aggregate quantified information systems of data non-locally. While able to mirror awareness to generate local access for conscious re-integration of information, it's important to note that the Internet, as a system, lacks the magnitudes of synthesis, as well as the multidimensional openness of conscious systems, which can process qualia-rich aesthetic information into emotional imprints, acts of will, creativity, or self-awareness. Google's latest AGI may dream in electric sheep, but for it to harness the force of consciousness enough to motivate standing in an uncomfortable line overnight for opening day tickets to the latest "Star Wars" film, or give its friend an intimate gift, would require transcendent interpretive functionality, mechanically operating in the quantum realms of resonant transactions of sensation, that conscious systems successfully absorb and preserve during "closed" or "semi-closed" states like sleep or death. 
Such desires and passions - excitement to see a new film, gratitude towards a friend, the wonder from a dream, awe from discovering something new-readily found in any character of Shakespeare or Chekov, are all aspects of an emotionally open mind's invitation to consider and act in relation with their own fate. Tragedy, comedy, or other narrative forms are not merely artifacts of culture, as Umberto Eco points out in The Open Work; but living, creative nodes shaping all of biological awareness' total field of perception, which can be sensed, integrated, and expressed with the comparatively robust biphasic, cognitive instrumentation of attention and resolution of awareness.

Similar to the example of encountering Pythagoras' Theorem, "The play's the thing wherein we'll catch the conscience of the king," is an aesthetic bundle for instance that regenerates meaning through its non-local, indeterminate openness to co-relative (i.e., indirect) cognitive architectures in interrelated quantum fields, and upon activation imprints within current waveforms of ZPF. In other words, when that line of text is received in some "meaningful" form by a reader or audience member, when it "plays," like a meme, the transmission of information causes a qualia-producing collapse, which potentially opens, or "unlocks," bundles of stored, or latent, co-relative cultural, psychological, physical, and biological information packets during integration. The chain reaction of integration may be indirectly networked, like "weak" forces or "weak" social contacts, as well as primarily subconscious, while diffusion plays out in the harmonics of various background fields.

With so many dimensions synthesizing during qualia, as well as imprinting upon non-local wave fields, Transhumanist speculations about sentient AGI becomes somewhat of a "batteries not included" ex machina wrapped in Frankenstein's monster, until algorithmic interplay can orchestrate coherency within the magnitudes greater "ocean" of harmonically-based, finely tuned, non-algorithmic, "real-time," dynamic possibilities - making their exits and their entrances amid life's symphony of perception. Humans can "float like a butterfly" or "sting like a bee" one moment, and "sing like a lark," or "cry like a donkey" the next because they've developed resonant perceptiveness to the Outernet of consciousness (a phenomenal network with oodles more bandwidth than the server bound variety), rather than formulating a perception based on mirroring perception.

On the other hand, the Internet's processing architectures, and quantified networking abilities may mirror the mirroring of biotic experience, but will not soon reflect on its experiences as a mirroring process; nor soon feel empathy or guilt over an illegally poached lion, rhino, or elephant. That's because its algorithms, however self-correcting, inevitably must bend back through the lenses of conscious interpretation in order to be integrated into that Outernet of biological perception enfolded and unfolding throughout Merleau-Ponty's phenomenological field (Merleau-Ponty, 2014). The Internet does however provide a huge amount of psychic feedback in analytical and social realms of collective cognitive trends, and will continue to develop into "holodeck-esque" representations of autonomy; but these projections are merely a new form of theatre for con- 
sciousness, rather than zygotes of new species.

When Hamlet tells Horatio "there are more things in heaven and earth, Horatio, than are dreamt of in your philosophy," he evokes a lullaby of the conscious imagination's openness to pure possibility. Even Hamlet, that prince of tongues, can only crack open a peanut shell of Shakespeare's sense of life's mysterious core-its tremendum. Iconic as they are his words merely suggest the way, make a Platonic dare, contemplate poetically, or indirectly glimpse at free will. Yet no machine will soon catch sight of such skills no matter it's power for computation.

So let Bostrom or Kurtzweil dream up their best critiques (Bostrom, 2014; Kurzweil, 2016). Digital is still a self-contained medium, which will for now reflect what we bring to it - like the manufactured shape of a knife, or the compositional structure of a popular music genre - evolving in alienation from complex conscious states like "inspiration" or "stuffy" where contemplative awareness comes into play (Greysen, Kind, \& Chretien, 2010). Our technology is alone with us, no matter how many new angles of our faces it can show or identify.

\section{Conclusion (Beauty in the Frame)}

If we consider that consciousness can create openings in the universe by organizing energy into complex biological structures capable of self-organizing qualia-rich communications, and their informational orders; life's integrative and compositional nature exists, however briefly, FAPP in hypothetically true defiance of Newton's 2nd Law-by decreasing disorder. Platonic values of mathematical and musical poetics prove relevant as epistemological guides to basic factors of resonance and radiance in relation to a common understanding of conscious mechanics, dynamics, and aesthetics—though of course Indian philosophers long ago affirmed phenomenological concepts of a cosmic holofield called "Akasha” (Laszlo, 2007). Luckily thinkers such as Eco and Merleau-Ponty have reminded science that, "The connection between essence and existence is not found in experience, but rather in the idea of the infinite" (Merleau-Ponty, 2014). This is an idea that researchers like Hameroff, Keppler, Atasoy, Kauffman, Bandyopadhyay, and others are now firmly digging into. To understand conscious systems wherein experience can freely oscillate through operant dynamics-stochastic, shared, and communicable-the entire system must be fundamentally open to constituting unprecedented possibilities within its modes of receptivity, integration, and transmission. The resonant and radiant factors internal and transcendent to the field dynamics of such systems would imprint in endless recombinations along encodings of perceivably recursive energetic and informational patterns, such as DNA and the anthropic principles, as well as within observable musical and organic geometric structures including octaves, the golden ratio, $\pi$ redundancy, genetic coding recursions and redundancy, Fourier series, prime number factorization, Fibonacci sequences, fractals, Laplace eigenfunctions, Bravyi and König's bound, and cellular structures in biology 
(Bravyi, Englbrecht, König, \& Peard, 2018).

The main contribution of this research is treating biological responsiveness in terms of a field dynamics with theoretical NCC, which effectively integrate the correlative observer and the phenomenon observed by composing energetic and informational phase shifts into higher level synthesis of critical mechanic and aesthetic signals into self-regulatory harmonic patterns (Atasoy et al., 2016; Peil, 2016). Within this field of play, CST establishes a harmonic-based discourse between the haecceity of possibilities, probabilities, awareness, attention, and qualia experiences as the key operants. By understanding the magnitudes of fundamental openness within natural communications networks, phenomena of consciousness can be correlated to irreducible receivers of perceptual attractors that contribute criticality to both homeostasis and epigenetics in living systems. During coherence, biological imprint increases systemic complexity through resonant phase locking that transfers disordered ZPF states to at least partially ordered qualia. Human cognitive structures, and their connectome harmonics, have adapted within this field to interpret for Platonic frequencies of meaningfulness, such as "truth," "beauty," or "good" as neuro-semantic representations of SED feedback from attractor-driven ZPF modification patterns. The resulting field dynamics creates a poetic framework for phenomenal classification of ZPF information states to "shed light on the internal structure of qualia space" (Keppler, 2016) by providing a co-relative epistemology for veracity. However, the space for further exploration is vast in articulating the energetic and informational impact of open mindedness in living systems' ability to effectively transmit conscious states nonlinearly and adaptively. Further support of this biolinguistic understanding of qualia space would benefit from experimental data that links ZPF modification to correlating resonance patterns of various conscious states, and their attractors.

\section{Energetic and Informational Impact of Open Mindedness in Living Systems}

Hameroff's research into the "Quantum Underground" of microtubules, pi-resonance cloud bonding, and solubility of anesthetics posits a coherence rate of $68 \mathrm{Thz}$ for conscious systems, which would accommodate physical responsiveness guidelines established by Ervin Laszlo's Integral-TOE, biological implications raised by Robert Lanza in Biocentrism, informational capabilities discussed in Tononi's IIT, the phenomenological considerations of Merleau-Ponty and Keppler's SED states, compositional and linguistic interpretations of Umberto Eco and Noam Chomsky, and constitutional neural correlates (NCC) imagined by Jonah Leherer in Proust was a Neuroscientist, and found in Atasoy and team's Laplace operator eigenfunctions, mapping one pattern into another in scalable growth holarchies (Merleau-Ponty, 2014; Eco, 1989; Chomsky, 1995; Laszlo, 2007; Lehrer, 2008; Tononi, 2008; Lanza \& Berman, 2010; Hameroff, 2016; Keppler, 2016; Wilbur, 2017). Speaking of agency, free will, and compositional properties of matter Laszlo writes, "Leading physicists such as Freeman 
Dyson and philosophers of the stature of Alfred North Whitehead asserted that elementary particles are endowed with a form and level of consciousness." "Matter in quantum mechanics," Dyson said, "is not an inert subject but an active agent.... It appears that mind, as manifested by the capacity to make choices, is to some extent inherent in every electron" (Laszlo, 2007).

Where Laszlo conceives, "In that case there is no categorical divide between mind and matter;" my mind bend's toward Chalmer's combination question about "how do the little minds at the fundamental level add up to big minds," (Chalmers, 2013) to create a nonzero threshold (i.e., biological homeostasis) of $+1 d$ in correlation with MDA analysis of the dynamic field coherence of conscious systems. Considering Hameroff's "Myer-Overton" analysis of living systems, and taking the ZPF as substrate as suggested by Keppler's SED-based theory of a universal mechanism for consciousness; we might consider in a 16-dimensional experimental system the probable amplitude coefficient $(C)$ of integrated information harmonics at superposition $(q !)$ in "qubits," approaches:

$$
\leq 16 d^{\text {? }} \text { or } \varphi^{q !}\left(d^{? n \leq 16}\right) / \int\left(U_{\text {corr }} \nabla R\right)=C
$$

for neural correlates of consciousness (NCC) of indeterminately coherent experience (i.e., structural homologies) (Watson, Campbell, Anwar, \& Browne, 2015).

Such theoretical experimentation illustrates how conscious systems could dynamically absorb, integrate, and transmit the kinds of aesthetic-rich phenomena human neurological experience ties together coherently (Reimann et al., 2017). The dynamic openness to interaction and interpretation between Penrosian moments of collapse in CST's model also indicates integrated resonance and radiance factors found in astronomical standards. Correlations can be made to account for absolute magnitude measurements for supernovae, CMB estimates of the Planck spacecraft's 2014 and 2015 observations of matter proportions (DE $=68.3 \%, \mathrm{DM}=26.8 \%$, and $\mathrm{BM}=4.9 \%$ ) baryon acoustic confirmation of cosmic acceleration, and OHD tracking of inflation ${ }^{3}$ (Planck Collaboration, 2015). The recalibration for syntropic transmission effectively integrates the position of an observer, as a measuring instrument within the system in a "constant state of free will," that can "fish" outside its "perceived" system via consciousness' aspect of "open mindedness." This native quantum leaping IPE includes the abilities to physically compose, encode, recall, contemplate, and play (i.e., freely interpret) through the phenomenon of correlative agency (Laszlo, 2007; Vannini, 2009). Merleau-Ponty describes this process of cohering, or corroborating, disjointed fields: "One phenomenon triggers another, not through some objective causality, such as the one linking together the events of nature, but rather through the sense it offers-there is a sort of operative reason, or a raison d etre that orients the flow of phenomena without being explicitly posited in any of them" (Merleau-Ponty, 2014). When evolutionarily localized, this flow state at the core of ${ }^{3}$ Less the cost of recalibrating our understanding of thermodynamics to allow quantum mechanical transfers of indeterminacy, non-locality, and entanglement to add new dimensions of information amplification, which adds richness to the orchestra of experience, via cognitive factors such as mirroring and matching to the Newtonian mechanics of attraction and acceleration. 
biological openness (i.e. receptivity), introduces the complex formational possibilities of integrated energy and information abundantly represented in living organisms. Evolution therefore provides evidence of how organisms can leverage entanglement processes not driven by entropic loops, so not subject to classical laws of acceleration or conductivity to perceivably, albeit temporarily, increase observable order across scales of the cosmological system. As Vannini writes, "These observations have lead to the hypothesis that the organization and evolution of living systems (tissues, nervous system, etc.) can be guided by attractors (causes placed in the future) in a similar way to that which happens in fractal geometry" (Vannini, 2009). Eco's studies reveal how this key structural homology phenomenon builds recursively into ever more complex systems of meaning structures in the development of lifeforms. This acknowledgement of biology's compositional transference of aesthetics, for instance in dreams or art, plays into archetypal psychologist Patricia Berry's understanding that, “The image is itself an irreducible and complete union of form and content, and for us cannot be considered apart from either. Image is both the content of a structure and the structure of a content" (Berry, 2008). These structures of aesthetics play out in both representations of thermodynamic and harmonic phase shifts as evidenced by variabilities in Boolean networks (Shmulevich \& Kauffman, 2004; Kauffman, 2012; England, 2013; Deacon, 2016; Atasoy et al., 2016; Bandyopadhyay, 2016; LeDoux \& Brown, 2017).

A conscious system, or mind, provides the mechanism for homeostatic regulatory chemical bounds that organizes radiance $\leftrightarrow$ resonance transfers across thermodynamic and harmonic thresholds through frequency oscillation operants, such as serotonin and dopamine in the human cortical structure. Resonance imprints are amplified by water, light, and other environmental elements at criticality events, producing effects found in Laplace eigenfunction patterns across living systems. Some patterns contribute to work energy, while those that cross into the wavefunction spectrum become transmittable (i.e. communicable) across the Outernet. The human brain is the most perceptive instrument in known existence for receiving the Outernet's harmonic signal spectrum. The signal spectrum remains coherent due to sustained aperiodic radiant and resonant projection from dynamic SED transfer states (i.e. harmonic phase shifts). These moments of collapse are the universe's open doors between information and energetic shifts, or quanta and qualia spaces for living systems connected by the Outernet.

These MDA considerations accounting for the significance of agency in the ebb and flow of entropy's tide, thereby allow organisms to make sense of their own possible learned and creative potentials in dynamic relation to determinant shaping positions of resolution, homeostasis, expression, leaping, beaming, dissolution, and zero-point within all coherent fields. When the mind's internal factors are treated as open contributors of meaning to the system, the aesthetics of starbursts or flowers blooming, as well as the harmonics of recurrent cosmic ratios, or Thomas Tallis motets, all bestow logical phenomenal compositions of 
energy and information, subject to aggregation into decreasing waves of disorder, dependent on perceive-ability and ease of communication (Schofield, 1951; Eco, 1989; Laszlo, 2007).

By framing our universe within a quantum biologically-backed "poetics of openness" a scientific shift towards understanding consciousness as a syntropic force, which through all other forces, including weak and/or gravitational interactions, composes matter "funda-mentally" perceptive to receiving, integrating, and responding through coherent field dynamics. Like the redshift phenomenon, the interaction of these dynamics indirectly reveals an aesthetic universe correlated to many effects of information and energetic events that impact qualia producing phenomena including:

radiance, resonance, dimension, structure, scale, frequency, range, coherence, directionality, perception, composition, formativity, pluralism, communicability, interpretability, reflection, and responsiveness...

In the amplituhedron of IIT information theory, these dimensions constitute the basic aesthetic effects on conscious systems' experiences of their reality, dynamized by Merleau-Ponty's phenomenal field, and mechanically confluent as our common yet subjective existence. It is the organizing, integrative, and interdependent flow, or force, of consciousness, which physically awakens remote spaces of "no collapse", to constitute new possibilities via localized compositional powers of integration, including cognition. Meanwhile, the great eye of Awareness opens the system to measurement by detection of probabilities, and coherent perception. By its very presence, open mindedness in conscious systems adapts the system (i.e., the Outernet) by making matter and interrelatedness meaningful via interpretable, responsive, significant acts of creation.

\section{Acknowledgements}

I thank Joachim Keppler, Stuart Hameroff, David Chalmers, Anirban Bandyopadhyay, Giulio Tononi, Robert Lanza, and Katherine Peil for their support.

\section{Conflicts of Interest}

The author declares no conflicts of interest regarding the publication of this paper.

\section{References}

Atasoy, S., Donnelly, I., \& Pearson, J. (2016). Human Brain Networks Function in Connectome-Specific Harmonic Waves. Nature Communications, 7, Article No. 10340. https://doi.org/10.1038/ncomms10340

Balázs, A. (2004). Is There a "Molecular Nirvana Principle"? Towards a Unified Resolutional Model of the Biological Symbol-Matter Dichotomy. Biosystems, 77, 1-10. https://doi.org/10.1016/S0303-2647(03)00101-1

Bandyopadhyay, A. (2016). Origin of Unique Pattern We Observe in the Resonance Frequency Distribution of Proteins. In Proceedings of Science of Consciousness Confe- 
rence. Tsukuba: Advanced Scanning Probe Micros, National Institute for Materials Science.

Bekoff, M. (1995). Cognitive Ethology, Vigilance, Information Gathering, and Representation: Who Might Know What and Why? Behavioural Processes, 35, 225-237. https://doi.org/10.1016/0376-6357(95)00050-X

Belluck, P. (2011). To Tug Hearts, Music First Must Tickle the Neurons. The New York Times.

Benzi, R., Sutera, A., \& Vulpiani, A. (1981). The Mechanism of Stochastic Resonance. Journal of Physics A: Mathematical and General, 14, L453. https://doi.org/10.1088/0305-4470/14/11/006

Berry, P. (2008). Echo's Subtle Body: Contributions to an Archetypal Psychology (pp. 64-69). Berlin: Spring Publications.

Bostrom, N. (2014). Superintelligence: Paths, Dangers, Strategies. Oxford: OUP.

Bravyi, S., Englbrecht, M., König, R., \& Peard, N. (2018). Correcting Coherent Errors with Surface Codes. NPJ Quantum Information, 4, 1-6. https://doi.org/10.1038/s41534-018-0106-y

Burns, S. P., Xing, D., \& Shapley, R. M. (2011). Is Gamma-Band Activity in the Local Field Potential of V1 Cortex a "Clock" or Filtered Noise? Journal of Neuroscience, 31, 9658-9664. https://doi.org/10.1523/JNEUROSCI.0660-11.2011

Burns, S. P., Xing, D., Shelley, M. J., \& Shapley, R. M. (2010). Searching for Autocoherence in the Cortical Network with a Time-Frequency Analysis of the Local Field Potential. Journal of Neuroscience, 30, 4033-4047. https://doi.org/10.1523/JNEUROSCI.5319-09.2010

Butler, J., Laclau, E., \& Žižek, S. (2000). Contingency, Hegemony, Universality: Contemporary Dialogues on the Left (pp. 230-233). Verso.

Carhart-Harris, R. (2016). Brain Imaging Studies with Psychedelic Drugs. In Proceedings of the Science of Consciousness Conference. London: Neuropsychopharmacology, Imperial College.

Chalmers, D. J. (1995). Facing Up to the Problem of Consciousness. Journal of Consciousness Studies, 2, 200-219.

Chalmers, D. J. (2013). The Combination Problem for Panpsychism.

Chomsky, N. (1995). Language and Nature. Mind, 104, 1-61. https://doi.org/10.1093/mind/104.413.1

Chomsky, N. (2007). Of Minds and Language. Biolinguistics, 1, 9-27.

Clobert, M., Saroglou, V., \& Hwang, K. K. (2015). Buddhist Concepts as Implicitly Reducing Prejudice and Increasing Prosociality. Personality and Social Psychology Bulletin, 41, 513-525. https://doi.org/10.1177/0146167215571094

Deacon, T. (2016). Evolution and Consciousness. Proceedings of the Science of Consciousness Conference, Tucson, Arizona, 29 April 2016.

Dillard, A. (1974). Pilgrim at Tinker Creek (pp. 151-81). New York: Harper's Magazine Press.

Dobbs, D. (2005). Zen Gamma. Scientific American Mind, 16, 9. https://doi.org/10.1038/scientificamericanmind0405-9b

Doesburg, S. M., Green, J. J., McDonald, J. J., \& Ward, L. M. (2009). Rhythms of Consciousness: Binocular Rivalry Reveals Large-Scale Oscillatory Network Dynamics Mediating Visual Perception. PLoS ONE, 4, e6142.

https://doi.org/10.1371/journal.pone.0006142 
Donini, P. (2010). Aristotle and Determinism.

Eccles, J. C. (2005). Evolution of the Brain: Creation of the Self (pp. 39-78). London: Routledge. https://doi.org/10.4324/9780203976661

Eco, U. (1989). The Open Work (pp. 10, 22, 26, 42, 48, 65, 82-84, 88, 90, 102-103, 141-143, 156, 176-179, 234-235).

Edwards, J. C. (2017). So It Was Microtubules after All? Journal of Consciousness Studies, 24, 24.

Emerson, R. W. (2004). Essays and Poems by Ralph Waldo Emerson. Barnes \& Noble Classics (pp. 189, 192). New York: Sterling Publishing.

England, J. L. (2013). Statistical Physics of Self-Replication. The Journal of Chemical Physics, 139, Article ID: 121923. https://doi.org/10.1063/1.4818538

Freeman, W. J. (2016). Quantitative Models for Field Dynamics of Cerebral Cortex Based in Ecog/Eeg. In Proceedings of the Science of Consciousness Conference. Berkeley, CA: Molecular and Cell Biology, University of California at Berkeley.

Grant, A., \& Dutton, J. (2012). Beneficiary or Benefactor Are People More Prosocial When They Reflect on Receiving or Giving? Psychological Science, 23, 1033-1039. https://doi.org/10.1177/0956797612439424

Greysen, S. R., Kind, T., \& Chretien, K. C. (2010). Online Professionalism and the Mirror of Social Media. Journal of General Internal Medicine, 25, 1227-1229. https://doi.org/10.1007/s11606-010-1447-1

Hameroff, S. (2014). Consciousness, Free Will and Quantum Brain Biology-The "Orch OR" Theory. In Quantum Physics Meets the Philosophy of Mind: New Essays on the Mind-Body Relation in Quantum-Theoretical Perspective (pp. 56, 99). Berlin: De Gruyter.

Hameroff, S. (2015). Darwin versus Deepak: Which Came First, Feelings or the Brain? The Huffington Post.

http://www.huffingtonpost.com/stuart-hameroff/darwin-versus-deepak-whic b 74810 $\underline{48 . h t m l}$

Hameroff, S. (2016). The "Meyer-Overton Quantum Underground" in Living Systems. Atmannspacher Harald.

Hameroff, S. R. (1999). Evolution and Function of Consciousness-Introduction. A Tree Tickles Its Branches, 8, 265.

Holmes, F., \& Danielson, C. (2012). Induction of Expanded States of Consciousness Using Spatial Angle Modulation ${ }^{T m}$ Audio Support Technology.

Hunicke, R., LeBlanc, M., \& Zubek, R. (2004). MDA: A Formal Approach to Game Design and Game Research. Proceedings of the AAAI Workshop on Challenges in Game AI, Vol. 4, 1722.

Kafatos, M., Tanzi, R. E., \& Chopra, D. (2011). How Consciousness Becomes the Physical Universe. Journal of Cosmology, 14, 3-14.

Kak, S., Chopra, D., \& Kafatos, D. (2014). Perceived Reality, Quantum Mechanics, and Consciousness. Cosmology, 18, 231-245.

Kanai, T., Minemoto, S., \& Sakai, H. (2005). Quantum Interference during High-Order Harmonic Generation from Aligned Molecules. Nature, 435, 470-474. https://doi.org/10.1038/nature03577

Kauffman, S. (2012). From Physics to Semiotics. Seminar in the Department of Semiotics, Tartu. http://www.uttv.ee/naita?id=11059

Kauffman, S. (2016). Mind-Body, Quantum Mechanics, Possibles and a Possible Panp- 
sychism. In Proceedings of the Science of Consciousness Conference. Seattle, WA: The Institute for Systems Biology.

Kauffman, S. A., \& Goodwin, B. C. (1990). Spatial Harmonics and Pattern Specification in Early Drosophila Development. Part II. The Four Colour Wheels Model. Journal of Theoretical Biology, 144, 321-345. https://doi.org/10.1016/S0022-5193(05)80079-7

Kaufman, S. B., Quilty, L. C., Grazioplene, R. G., Hirsh, J. B., Gray, J. R., Peterson, J. B., \& DeYoung, C. G. (2015). Openness to Experience and Intellect Differentially Predict Creative Achievement in the Arts and Sciences. Journal of Personality, 82, 248-258. https://doi.org/10.1111/jopy.12156

Keppler, J. (2013). A New Perspective on the Functioning of the Brain and the Mechanisms behind Conscious Processes. Frontiers in Psychology, 4, 242.

https://doi.org/10.3389/fpsyg.2013.00242

Keppler, J. (2016) On the Universal Mechanism Underlying Conscious Systems and the Foundations for a Theory of Consciousness. Open Journal of Philosophy, 6, 346-367. https://doi.org/10.4236/ojpp.2016.64034

Kohri, K., Lin, C. M., \& Matsuda, T. (2014). Scale-Dependent CMB Asymmetry from Primordial Configuration. Journal of Cosmology and Astroparticle Physics, 2014, 26. https://doi.org/10.1088/1475-7516/2014/08/026

Kozhevnikov, M., Elliott, J., Shephard, J., \& Gramann, K. (2013). Neurocognitive and Somatic Components of Temperature Increases during g-Tummo Meditation: Legend and Reality. PLoS ONE, 8, e58244. https://doi.org/10.1371/journal.pone.0058244

Kurzweil, R. (2016). The Singularity Is Near. In Ethics and Emerging Technologies (p. 393). Berlin: Springer. https://doi.org/10.1057/9781137349088 26

Lanza, R., \& Berman, B. (2010). Biocentrism: How Life and Consciousness Are the Keys to Understanding the True Nature of the Universe. Chapter 18: Consciousness Is a Fundamental Force. Dallas, TX: BenBella Books.

Laszlo, E. (2007). Science and the Akashic Field: An Integral Theory of Everything (pp. 25, 31, 35-37, 39-51, 63-70, 91, 118-122). Rochester, VT: Inner Traditions/Bear \& Co.

LeDoux, J. E., \& Brown, R. (2017). A Higher-Order Theory of Emotional Consciousness. Proceedings of the National Academy of Sciences, 114, Article ID: 201619316.

https://doi.org/10.1073/pnas.1619316114

Lehrer, J. (2008). Proust Was a Neuroscientist. Boston, MA: Houghton Mifflin Harcourt.

Leopold, A. (1949). A Sand County Almanac (pp. 104-08). New York: Oxford University press.

Lutz, A., McFarlin, D. R., Perlman, D. M., Salomons, T. V., \& Davidson, R. J. (2013). Altered Anterior Insula Activation during Anticipation and Experience of Painful Stimuli in Expert Meditators. Neuroimage, 64, 538-546.

https://doi.org/10.1016/j.neuroimage.2012.09.030

MacKenzie, S. B. (1986). The Role of Attention in Mediating the Effect of Advertising on Attribute Importance. Journal of Consumer Research, 13, 174-195. https://doi.org/10.1086/209059

Mattson, M. P. (2014). Superior Pattern Processing Is the Essence of the Evolved Human Brain. Frontiers in Neuroscience, 8, 265. https://doi.org/10.3389/fnins.2014.00265

McCrae, R. R. (1996). Social Consequences of Experiential Openness. Psychological Bulletin, 120, 323. https://doi.org/10.1037/0033-2909.120.3.323

Merleau-Ponty, M., \& Landers, D. A. (2014). Phenomenology of Perception (pp. xxv-xxviii, xxxii-xxxv, 9-12, 17-20, 23-27, 29-34, 39-47, 49-65). London: Routledge.

Mitchell, C. (2013). Experience and Interpretation: Comment on Ferreira. Australian 
Journal of Parapsychology, 13, 63.

Nystrom, J. F. (2004). On the Omni-Directional Emergence of Form in Computation. In International Conference on Cellular Automata (pp. 632-641). Berlin: Springer. https://doi.org/10.1007/978-3-540-30479-1 65

Olshausen, B. A., Anderson, C. H., \& Van Essen, D. C. (1993). A Neurobiological Model of Visual Attention and Invariant Pattern Recognition Based on Dynamic Routing of Information. Journal of Neuroscience, 13, 4700-4719.

https://doi.org/10.1523/JNEUROSCI.13-11-04700.1993

Oppenheimer, J. R. (1949). “The Open Mind.” The Atlantic. http://www.theatlantic.com/magazine/archive/1949/02/the-open-mind/305431 https://doi.org/10.1080/00963402.1949.11457024

Passingham, R. E., \& Wise, S. P. (2012). The Neurobiology of the Prefrontal Cortex: Anatomy, Evolution, and the Origin of Insight (No. 50). Oxford: Oxford University Press. https://doi.org/10.1093/acprof:osobl/9780199552917.001.0001

Penrose, R. (1999). The Emperor's New Mind: Concerning Computers, Minds, and the Laws of Physics (pp. 296-298). Oxford Paperbacks.

Penrose, R., \& Hameroff, S. (2011). Consciousness in the Universe: Neuroscience, Quantum Space-Time Geometry and Orch OR Theory. Journal of Cosmology, 14, 1-17.

Perunov, N., Marsland, R. A., \& England, J. L. (2016). Statistical Physics of Adaptation. Physical Review X, 6, Article ID: 021036. https://doi.org/10.1103/PhysRevX.6.021036

Peil, K. (2016). Emotional Sentience and the Nature of Phenomenal Experience. In Proceedings of the Science of Consciousness Conference. Kirkland, WA: EFS International.

Planck Collaboration (2015). Planck 2015 Results. XIII. Cosmological Parameters.

Raju, P. S. (1980). Optimum Stimulation Level: Its Relationship to Personality, Demographics, and Exploratory Behavior. Journal of Consumer Research, 7, 272-282. https://doi.org/10.1086/208815

Reimann, M. W., Nolte, M., Scolamiero, M., Turner, K., Perin, R., Chindemi, G., Markram, H. et al. (2017). Cliques of Neurons Bound into Cavities Provide a Missing Link between Structure and Function. Frontiers in Computational Neuroscience, 11, 48. https://doi.org/10.3389/fncom.2017.00048

Rokeach, M. (1960). The Open and Closed Mind.

Sarkar, S., \& Bhattacharyay, A. (2014). Emergent Gravity by Tuning the Effective Planck Length in Bose-Einstein Condensate (No. arXiv: 1405.1865).

Schirmer, A., Teh, K. S., Wang, S., Vijayakumar, R., Ching, A., Nithianantham, D., Cheok, A. D. et al. (2011). Squeeze Me, But Don't Tease Me: Human and Mechanical Touch Enhance Visual Attention and Emotion Discrimination. Social Neuroscience, 6, 219-230. https://doi.org/10.1080/17470919.2010.507958

Schneider, S. (2008). Future Minds: Transhumanism, Cognitive Enhancement and the Nature of Persons.

Schofield, B. (1951). The Manuscripts of Tallis's Forty-Part Motet. The Musical Quarterly, 37, 176-183. https://doi.org/10.1093/mq/XXXVII.2.176

Shakespeare, W. (1904). The Tragedy of Hamlet (p. 129). Cambridge, England: University Press.

Shmulevich, I., \& Kauffman, S. A. (2004). Activities and Sensitivities in Boolean Network Models. Physical Review Letters, 93, Article ID: 048701.

https://doi.org/10.1103/PhysRevLett.93.048701 
Tononi, G. (2008). Consciousness as Integrated Information: A Provisional Manifesto. The Biological Bulletin, 215, 216-242. https://doi.org/10.2307/25470707

Tononi, G., \& Koch, C. (2015). Consciousness: Here, There and Everywhere? Philosophical Transactions of the Royal Society B, 370, Article ID: 20140167.

https://doi.org/10.1098/rstb.2014.0167

Vannini, A. (2009). A Syntropic Model of Consciousness. Syntropy Journal, 2009, 100-138.

Ward, L. M., MacLean, S. E., \& Kirschner, A. (2006). Stochastic Resonance Modulates Neural Synchronization within and between Cortical Sources. PLoS ONE, 5, e14371. https://doi.org/10.1371/journal.pone.0014371

Watson, F. H., Campbell, E. T., Anwar, H., \& Browne, D. E. (2015). Qudit Color Codes and Gauge Color Codes in All Spatial Dimensions. Physical Review A, 92, Article ID: 022312. https://doi.org/10.1103/PhysRevA.92.022312

White, F. M. (1999). Fluid Mechanics. Boston, MA: McGraw-Hill.

Wilbur, K. (2017). Trump and a Post-Truth World: An Evolutionary Self-Correction (pp. 53-57). Deep Dive Ebook: Integral Life.

Wilson, E. (1961). Axel's Castle (p. 178). London: Collins, Fontana Library.

Wolchover, N. (2014). A New Physics Theory of Life. Quanta Magazine. https://www.quantamagazine.org/20140122-a-new-physics-theory-of-life

Wolchover, N. (2017). Physicists Uncover Geometric “Theory Space”. Quanta Magazine. https://www.quantamagazine.org/20170223-bootstrap-geometry-theory-space

Zizek, S. (2002). Lacan between Cultural Studies and Cognitivism. In J. Glynos, \& Y. Stavrakakis (Eds.), Lacan and Science (pp. 291-320). London: Karnac Press. https://doi.org/10.4324/9780429476501-12

Zohar, D., \& Marshall, I. N. (1990). The Quantum Self (p. 94). 